\title{
PHYTOSOCIOLOGICAL EXAMINATION OF IHLARA SPECIAL ENVIRONMENTAL CONSERVATION ZONE
}

\author{
KAYA, Ö. F. ${ }^{1 *}-$ CANSARAN, A. $^{2}$ \\ ${ }^{1}$ Department of Biology, Science and Art Faculty, Harran University, \\ P.O. Box 63190 Şanlıurfa, Turkey \\ ${ }^{2}$ Department of Elementary Education, Education Faculty, Amasya University, \\ P.O. Box 05100 Amasya, Turkey \\ *Corresponding author \\ e-mail: phytosociologist@gmail.com \\ (Received 27 $7^{\text {th }}$ March 2015; accepted $18^{\text {th }}$ Sept 2015)
}

\begin{abstract}
The aim of this study was to detect the syntaxa in the Ihlara valley vegetation which have not been previously phytosociologically assessed. Ihlara Valley is geographically located in Central Anatolia and in the Irano-Turanian phytogeographical regions. The study area is located within the B5 square based on the Grid System (Davis et al. 1965-1985). According to the Braun-Blanquet (1932) method, there are three plant associations belonging to three different vegetation types in the region; Pistacio terebinthi - Celtidetum tournefortii (shrub vegetation), Astragaletum karamasici - microcephali (tragacanth steppe vegetation), and Rubo sancti - Salicetum albae (riparian vegetation). These three syntaxa are all new to the world of science.
\end{abstract}

Keywords: Ihlara Valley, Central Anatolia, syntaxonomy, Aksaray, Turkey

\section{Introduction}

A majority of the phytogeographers suggest that Central Anatolia, which includes Ihlara Valley, forms the western border of Central Asian steppe and desert strip; in other words, the Irano-Turanian floristic region. Walter (1962) qualifies Central Anatolia as a steppe along with Syria, Mesopotamia and Eastern Anatolia. Additionally, Walter (1972) explains that Central Anatolia is not a semi-desert but a steppe where annual crops and geophytes are quite common (Çetik, 1985).

The Central Anatolian plains, mountains excluded, is a vast plateau with an altitude ranging from 800-1600 m (Çetik, 1985). In the Central Anatolia Region, summers are hot and dry and winters are harsh and long. The climatic characteristics are of a continental climate in terms of temperature conditions and steppe climate in terms of rainfall conditions. The once-present forests were significantly destroyed throughout the millennia of human habitation (Günal, 2013). Central Anatolia has been intensely inhabited since almost as early as 3000 BC. Needs for firewood, construction timber, mining operations, overgrazing, and deforestation for agricultural lands have formed the current anthropogenic Central Anatolian steppe by eliminating the natural vegetation. Therefore, the borders of the real steppe in Central Anatolia have enlarged and the neighboring low and high mountain steppes have emerged (Çetik, 1985). Larch, oak and juniper species are conserved in forest residues in the higher parts of the region (Avc1, 2005).

In the Central Anatolian plain areas, which include the upper parts of Ihlara Valley, plant taxa belonging to the anthropogenic steppe vegetation, consisting generally of xerophytes, are common. Additionally, there are taxa typical of shrub and riparian 
vegetation in the micro-climate area (slopes and bottom) formed by the Melendiz River, due to Ihlara Valley being a narrow canyon with a depth of approximately $100 \mathrm{~m}$.

Ihlara Valley, one of the Turkey's most important historical and natural beauties located in Cappadocia, was formed results of millions of years of geological events and thousands of years of cultural accumulation. Geomorphologic structure, volcanism, hydrothermal activity and cultural activities can be counted among the factors that influenced the formation of natural vegetation. Ihlara Valley, in which anthropogenic steppe characterized the great plains of Central Anatolia, is a kind of microclimate of an area of an island with its own characteristic vegetation.

The Cappadocia region and the surrounding area, including Ihlara Valley, is regarded as a moderately-well characterized region in terms of its floristic aspects (Çırpıc1, 1987). Several phytosociological studies on various vegetation types have been carried out (Düzenli, 1976; Aydoğdu et al., 1999; Ketenoğlu et al., 2000; Aydoğdu et al., 2002; Baysal, 2003; Aydoğdu et al., 2004; Ketenoğlu et al., 2008).

In this study the natural vegetation of Ihlara Valley, declared as Special Environmental Conservation Zone and protected since 1990 to conserve its cultural assets and biodiversity, is aimed to be determined phytosociologically. This study is hoped to contribute to the future studies aiming to investigate natural succession of this area by time.

\section{Materials and Methods}

Thirty relevés were taken from the vegetation types in the study area which represent the vegetation in terms of floristic composition and were adequately homogeneous. The relevés were the "minimal area" representing the vegetation, where possible. The width of the relevés in the study area was determined as $400 \mathrm{~m}^{2}$ for shrub vegetation, $25 \mathrm{~m}^{2}$ for tragacanth steppe vegetation and $50 \mathrm{~m}^{2}$ for riparian vegetation.

Evaluation of floristic table in terms of plant sociology was performed using the Braun-Blanquet (1932) method and Frey \& Lösch (1998) detailed abundance-cover scale were used. According to this scale; $r=1$ individual (also rare outside the relevé, small plant); + = 2-5 (small) individuals, cover $<5 \% ; 1=6-50$ individuals, cover $<5 \%$; or few larger individuals (often given as 1-5) with a cover up to $5 \% ; 1 \mathrm{~m}=$ many individuals ( $>50)$, cover $<5 \% ; 2 \mathrm{a}=$ cover $5-12.4 \% ; 2 \mathrm{~b}=$ cover $12.5-25 \% ; 3=$ cover $25-50 \% ; 4=$ cover $50-75 \% ; 5=$ cover $75-100 \%$.

Syntaxa belonging to the shrub vegetation were classified according to BraunBlanquet et al. (1952), Barbero and Quézel (1976), Quézel et al. (1980), and Akman (1995); syntaxa belonging to the step formations were classified according to Quézel (1973) and Akman et al. (1984; 1985) and syntaxa belonging to the riparian vegetation were classified according to Rivas-Martínez (1991). Phytosociological nomenclature codes (Weber et al., 2000) were used for naming the sociological units.

The comparison of described syntaxa with the similar associations regarding the floristic composition was made by using Sørensen (1948) similarity formula:

$$
\mathrm{C}_{\mathrm{s}}=(2 \times \mathrm{C} \times 100) /(\mathrm{A}+\mathrm{B})
$$

$\left(C_{S}=\right.$ similarity coefficient, $A$ is the number of species found in site $A, B$ is the number of species in site $\mathrm{B}$ and $\mathrm{C}$ is the number of species shared by the two sites). 
Analysis of representative soil samples taken from the study area were performed at Soil and Fertilizer Research Institute. EC, $\mathrm{pH}$ and salt were measured from the extract obtained from soil saturation paste (Richards, 1954); soil particle size distribution according to Bouyoucos method (Bouyoucos, 1936); available lime in soil according to Calsimeter method (Sherrod et al., 2002); available phoshorous in soil according to Olsen method (Olsen et al., 1954); available potassium according to Ammonium acetate method (Schollenberger and Simon, 1945); soil organic matter according to modified Walkley-Black method (Walkley and Black, 1934).

In order to define the climatic characteristics of the study area, data from Aksaray meteorological station were obtained from the General Directorate of Meteorology Affairs (Anonymous, 2010). In order to determine the bioclimatic zones,

$$
\mathrm{Q}=2000 . \mathrm{P} / \mathrm{M}^{2}-\mathrm{m}^{2}
$$

developed for Mediterranean Region and,

$$
\mathrm{S}=\mathrm{PE} / \mathrm{M}
$$

formula, developed to describe dry seasons (Emberger, 1954), were used along with studies by Akman and Daget (1971) and Akman (1999).

Studies by Davis (1965-1985), Davis et al. (1988), and Güner et al. (2000) were used for the identification of collected taxa.

\section{Study Site}

Ihlara Valley, which was defined as the Ihlara Special Environmental Conservation Zone in 1990, is approximately $40 \mathrm{~km}$ southeast of the province of Aksaray, close to Güzelyurt district, covering an area of $54.64 \mathrm{~km}^{2}$ at the intersection point of $34^{0} 16^{\prime} 00^{\prime \prime}$ latitude and $38^{0} 15^{\prime} 00^{\prime \prime}$ longitude. The region is completely a natural and archaeological site with a narrow $(50-500 \mathrm{~m})$ and a deep $(20-110 \mathrm{~m})$ structure formed by Melendiz Stream which creates an oasis in an arid region. Ihlara Valley attracts attention with its unique geological and hydrogeological structure. The valley floor, which is located at an altitude of $1220 \mathrm{~m}$, is covered with rocks as a result of the volcanic activities of Hasan Mountain and Erciyes Mountain during the Pliocene geological era (Anonymous, 2012). The deep valleys and geological units in this valley were formed over a period of about 5 million years. Ihlara valley is located in the Tuz Gölü (Salt Lake) basin in the Central Anatolia region, which is surrounded by North Anatolia (Black Sea) to the north, Southern Anatolia (Taurus) Mountains to the south, the East Anatolian high plateau to the east and the Central Western Anatolia high plateau to the west (Gülkal, 1999). Ihlara Valley, formerly known as "Peristremma", is $14 \mathrm{~km}$ long (Anonymous, 2012), including the towns of Ihlara and Selime and Yaprakhisar and Belisirma villages. The valley is connected to Güzelyurt district at the foothills of Hasan Mountain (3268 m) and Melendiz Mountain (2963 m) and extends towards the northwest (Gülkal, 1999). The Melendiz River (this river was called Potamas Kapadokus, meaning Cappadocia River, in primeval days) extends from one end of the valley to the other. Ihlara valley forms a part of the Cappadocia region with a history stretching back to 3000 BC. Many churches carved from the rock during the Roman Empire era at the 4th century representing the artistic styles of the period can still be found in the valley (Anonymous, 2012) (Fig. 1). 


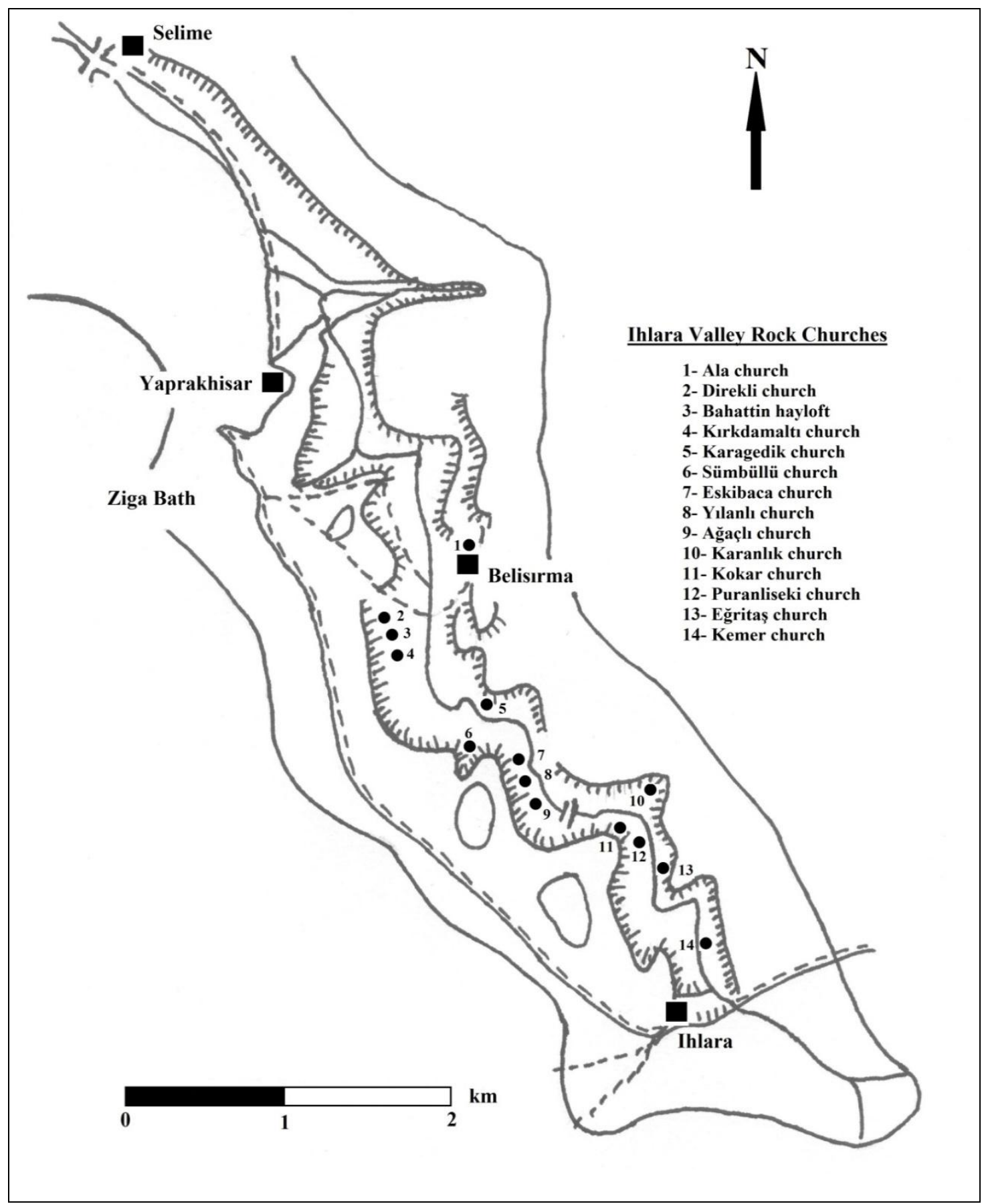

Figure 1. Topographic map of the study area (Tanker et al. 1989)

A large part of study area consists of volcanic rocks from the Miocene, Pliocene and Quaternary eras. All of these units are covered with travertine, talus and alluvium. The main factor determining the region's morphology and geological activities is erosion, which has continued intensively to the present day. Hasan Mountain, which is very close to the valley, was formed during Paleozoic and Mesozoic eras. Despite the increase during heaves formed during Neogene (Late Tertiary) and Quaternary eras, basins have remained relatively low. As a result of tectonic movements, which caused the eruption of Hasan Mountain volcano, a wide range volcanic layers has covered the 
surrounding surfaces. Natural hot water gushing from the fault line caused by pressure and temperature during the same movements led to the formation of the Ziga spas between Yaprakhisar and Ihlara towns (Anonymous, 2012).

Volcanic activity occurred most recently in the Quaternary era, thus the area is geologically quite young. Therefore, the original features of the geological structure have survived intact to the present day. Erosion caused by the tributaries of the Melendiz River along with other climatic conditions have given Ihlara Valley and the close vicinity their present appearance. The non-horizontal position of the geological units indicates that major tectonic activities have given a gradient to the region (Gülkal, 2005) (Fig. 2).

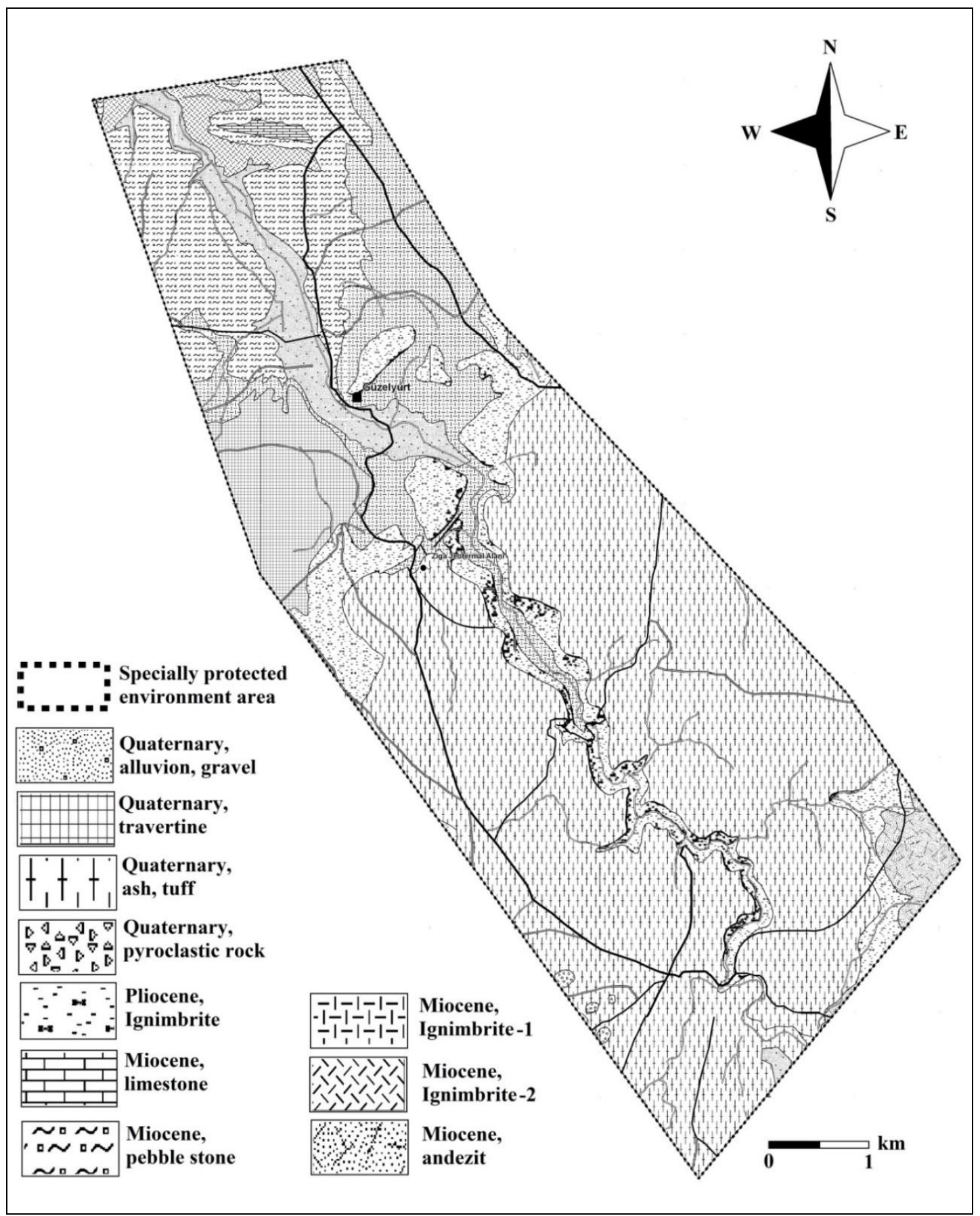

Figure 2. Geology map of Ihlara valley 
In Ihlara Valley, tuff stones, which were formed as a result of volcanic eruptions, deeply affect the structural character of the environment, having been worn by wind, erosion and other natural factors to form "Fairy Chimneys" of various shapes and colors between Selime and Yaprakhisar. Tectonic movements have collapsed the areas covered with coarse grains and crumbly rocks in which tuff stones are dominant at some places, while gray, green and brown colors are found at others. The Melendiz River, flowing along the Ihlara Valley, has gained a greater depth by excavating the bottom of the canyon valley, which was formed as a result of such a collapse (Gülkal, 1999).

\section{Results}

As a result of the study, syntaxa belonging to shrub, tragacanth steppe and riparian vegetation types (plant association) were determined with the syntaxonomic units that they belong to. The three identified syntaxa are new to the world of science.

\section{Pistacio terebinthi - Celtidetum tournefortii ass. nova (Table 1)}

Holotypus: relevé 3, Karagedik church and its surrounding, Cover \%85, $400 \mathrm{~m}^{2}$ Lat: $38^{\circ} 15^{\prime} 42^{\prime \prime} \mathrm{N}$, Lon: $034^{\circ} 17^{\prime} 35^{\prime \prime} \mathrm{E}, 1193$ m.

This association flourishes on volcanic bedrock. Texture type of the soil is clay loam and clay, containing 1.51-8.60\% organic substances. The soil on which the association flourishes shows mildly alkaline and mildly acidic reactions $\left(\mathrm{pH}\right.$ 6.59-7.76). $\mathrm{CaCO}_{3}$ percentage is $0.89 \%$ and saturation is $61-98 \%$. Total salt content varies between 0.026 and 0.077 .

The association consists of two vegetation types including shrubs and herbs. It flourishes at locations where the slope is $15^{\circ}-40^{\circ}$. Shrub overlap is between $70-85 \%$ with an average height of 3 meters. Grass overlap is between $50-60 \%$ with an average height of $25 \mathrm{~cm}$. The association was found between the 1180th and 1205th meters of the study area.

Physiognomy of the association is dominated by Celtis tournefortii; this taxon shows a wide distribution throughout Turkey. The co-dominant Pistacia terebinthus subsp. terebinthus is an Irano-Turanian element. Malva sylvestris, Crupina crupinastrum, Vicia peregrina, Lathyrus cicera, Matthiola longipetala subsp. bicornis, Trigonella aurantiaca, Medicago sativa subsp. sativa, and, Stachys cretica subsp. anatolica are frequently found to participate in the association.

\section{Astragaletum karamasici - microcephali ass. nova (Table 2)}

Holotypus: relevé 17, Belisırma locality, Cover \%85, $25 \mathrm{~m}^{2}$ Lat: $38^{\circ} 16^{\prime} 03^{\prime \prime} \mathrm{N}$, Lon: $034^{\circ} 17^{\prime} 06^{\prime \prime} \mathrm{E}, 1241 \mathrm{~m}$.

This association flourishes on volcanic bedrock. Texture type of the soil is loam, containing $0.38-0.48 \%$ organic substances. The soil on which the association flourishes shows mildly alkaline reactions $(\mathrm{pH} 7.99-8.01) . \mathrm{CaCO}_{3}$ ratios varies between 1.19$1.33 \%$ and the saturation is $44-47 \%$. The total amount of salt is 0.018 to 0.020 .

The association was detected where the slope was $10^{\circ}-30^{\circ}$. There are no tree or shrub layers in vegetation structure, consisting exclusively of grass layer. Grass overlap is between $70-85 \%$. The average grass height is $35 \mathrm{~cm}$. 
Table 1. Pistacio terebinthi-Celtidetum tournefortii ass. nova

\begin{tabular}{|c|c|c|c|c|c|c|c|c|c|c|c|c|}
\hline & Relevé No & 1 & 2 & 3 & 4 & 5 & 6 & 7 & 8 & 9 & 10 & \\
\hline & Size of plot $\left(\mathrm{m}^{2}\right)$ & 400 & 400 & 400 & 400 & 400 & 400 & 400 & 400 & 400 & 400 & \\
\hline & Parent rock & Vol & Vol & Vol & Vol & Vol & Vol & Vol & Vol & Vol & Vol & \\
\hline & Inclination $\left({ }^{\circ}\right)$ & 25 & 20 & 30 & 30 & 15 & 25 & 35 & 30 & 40 & 40 & \\
\hline & Altitude (m) & 1185 & 1180 & 1193 & 1188 & 1190 & 1205 & 1203 & 1180 & 1185 & 1197 & $\underset{\mathscr{E}}{=}$ \\
\hline & Exposition & $\mathrm{NE}$ & $\mathrm{NE}$ & $\mathbf{N E}$ & $\mathrm{E}$ & $\mathrm{E}$ & W & $\mathrm{W}$ & $\mathrm{NE}$ & $\mathrm{NE}$ & $\mathrm{NE}$ & 遇 \\
\hline & Coverage (\%) & 80 & 85 & 85 & 70 & 70 & 75 & 75 & 80 & 80 & 80 & 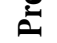 \\
\hline \multicolumn{13}{|c|}{ LF } \\
\hline & \multicolumn{12}{|c|}{ Differential and characteristic species of the association } \\
\hline $\mathrm{Ph}$ & Celtis tournefortii & 5 & 5 & 5 & 4 & 4 & 4 & 4 & 5 & 4 & 5 & $\mathrm{~V}$ \\
\hline $\mathrm{Ph}$ & Pistacia terebinthus subsp. terebinthus & + & 1 & 1 & 1 & 1 & + & + & 1 & + & 1 & $\mathrm{~V}$ \\
\hline \multicolumn{13}{|c|}{ Characteristic species of Melitto albidae-Quercion frainetto } \\
\hline $\mathrm{Ph}$ & \multicolumn{12}{|c|}{ Characteristic species of Querco cerridis-Carpinetelia orientalis } \\
\hline $\mathrm{Ph}$ & Colutea cilicica & 1 & + & 1 & & 1 & + & + & & 1 & & IV \\
\hline $\mathrm{H}$ & Tanacetum parthenium & + & & + & 1 & & & + & & + & & III \\
\hline $\mathrm{Ph}$ & Pyracantha coccinea & & + & & & + & & & & + & + & II \\
\hline $\mathrm{H}$ & $\begin{array}{l}\text { Characteristic species of Quercetea pul } \\
\text { Coronilla varia } \text { L. subsp. varia } \\
\text { Companions }\end{array}$ & scenti & & + & 1 & + & & & + & & & II \\
\hline $\mathrm{H}$ & Malva sylvestris & 1 & & 1 & + & 1 & 1 & & + & & + & IV \\
\hline Th & Crupina crupinastrum & 1 & & + & 1 & & 1 & & 1 & + & & III \\
\hline Th & Vicia peregrina & 1 & & + & + & 1 & & & & + & + & III \\
\hline Th & Lathyrus cicera & + & & & + & & 1 & & 1 & & + & III \\
\hline Th & Matthiola longipetala subsp. bicornis & & & 1 & 1 & & 1 & & & 1 & 1 & III \\
\hline Th & Trigonella aurantiaca & & & 1 & + & & & + & + & 1 & & III \\
\hline $\mathrm{H}$ & Medicago sativa subsp. sativa & + & & & + & + & & + & & & + & III \\
\hline $\mathrm{H}$ & Stachys cretica subsp. anatolica & + & + & & + & + & + & & + & & & III \\
\hline $\mathrm{H}$ & Trifolium pratense var. pratense & & + & 1 & 1 & & & 1 & 1 & & + & III \\
\hline Th & Scabiosa rotata & 1 & 1 & & & + & + & & 1 & & 1 & III \\
\hline $\mathrm{H}$ & Parietaria judaica & & + & + & 1 & & & 1 & + & & & III \\
\hline $\mathrm{H}$ & Valeriana dioscoridis & & + & & & + & & + & + & + & & III \\
\hline $\mathrm{H}$ & Onosma aucheranum & + & & + & & + & + & & & + & + & III \\
\hline $\mathrm{Ph}$ & Amygdalus orientalis & & + & + & + & & & + & & & + & III \\
\hline $\mathrm{H}$ & Umbilicus erectus & & + & + & & + & & + & & & & II \\
\hline $\mathrm{H}$ & Fibigia eriocarpa & & & + & & + & & + & & & + & II \\
\hline $\mathrm{H}$ & Salvia cilicica & & 1 & $\mathbf{1}$ & & 1 & & & & & + & II \\
\hline Th & Logfia arvensis & + & & & & + & & & + & & & II \\
\hline Th & Myrrhoides nodosa & & + & + & & & & & & + & & II \\
\hline $\mathrm{Ph}$ & Rosa canina & & & & & + & + & & 1 & & & II \\
\hline $\mathrm{H}$ & Urtica dioica & & & + & + & & & + & & & + & II \\
\hline $\mathrm{H}$ & Silene compacta & + & & + & & & + & & & + & & II \\
\hline $\mathrm{Ph}$ & Crateagus monogyna subsp. monogyna & & + & + & & + & & & + & & & II \\
\hline $\mathrm{Ph}$ & Cotoneaster nummularia & & + & + & & & & & + & & & II \\
\hline $\mathrm{H}$ & Galega officinalis & & & + & & & + & & + & & & II \\
\hline $\mathrm{H}$ & Arctium minus subsp. pubens & & & + & & 1 & + & & & & & II \\
\hline $\mathrm{H}$ & Silene swertiifolia & & & + & & & + & & + & + & & II \\
\hline Th & Vicia villosa subsp. dasycarpa & & + & & + & & & + & & & + & II \\
\hline $\mathrm{H}$ & Orthurus heterocarpus & & & + & + & & + & & & + & & II \\
\hline Th & Asperula arvensis & & + & & + & & 1 & & & 1 & & II \\
\hline $\mathrm{H}$ & Salvia tomentosa & 1 & & $\mathbf{1}$ & & & & + & & & & II \\
\hline $\mathrm{H}$ & Salvia verticillata subsp. verticillata & & + & & & 1 & & + & & & & II \\
\hline $\mathrm{Ph}$ & Pyrus bulgarica & & + & & & + & & + & & & & II \\
\hline $\mathrm{H}$ & Geranium pyrenaicum & & & + & & & + & & & & + & II \\
\hline G & Eremurus spectabilis & + & & + & & & & + & & + & & II \\
\hline $\mathrm{H}$ & Tripleurospermum oreades var. oreades & & + & & + & & & & + & & + & II \\
\hline Th & Vicia cuspidata & & 1 & & & + & & + & & & 1 & II \\
\hline $\mathrm{H}$ & Symphytum bornтиelleri & + & & + & + & & & + & & & & II \\
\hline $\mathrm{H}$ & Sanguisorba minor subsp. muricata & & & + & & & + & & & + & & II \\
\hline
\end{tabular}


Table 1. Continued

\begin{tabular}{|c|c|c|c|c|c|c|c|c|c|c|c|}
\hline Th Cnicus benedictus var. benedictus & + & & & + & & + & & & & & II \\
\hline Th Vicia grandiflora var. grandiflora & & & + & & & & + & & & + & II \\
\hline $\mathrm{Ph}$ Crataegus meyeri & + & & & + & & & $\mathrm{r}$ & & & & II \\
\hline $\mathrm{H}$ Bryonia alba & & + & & + & & + & & & + & & II \\
\hline H Geum urbanum & + & & + & & & + & & + & & & II \\
\hline H Asyneuma lobelioides & & & + & & & + & + & & + & & II \\
\hline H Torilis ucranica & & + & & + & & & & + & & & II \\
\hline Th Delphinium peregrinum & & + & & + & & & + & & 1 & & II \\
\hline $\mathrm{Ph}$ Pistacia vera & + & & + & & & & + & & & & II \\
\hline H Smyrnium cordifolium & & & & + & + & & & & & & I \\
\hline Ph Rhamnus oleoides subsp. graecus & & & & + & & & & + & & & I \\
\hline $\mathrm{Ph}$ Amygdalus $x$ balansae & & + & & & & + & & & & & I \\
\hline $\mathrm{H}$ Chelidonium majus & & + & & & & & + & & & & I \\
\hline G Ornithogalum orthophyllum & & & + & & & & + & & & & I \\
\hline
\end{tabular}

Astragalus microcephalus, which determines the physiognomy of the association, is an Irano-Turanian element which shows a vast distribution in steppes in Turkey. Codominant Astragalus karamasicus is an endemic taxa generally native to the Central Anatolia. Xeranthemum annuum, Teucrium polium, Marrubium globosum subsp. globosum, Ziziphora taurica subsp. taurica, Thymbra sintenisii subsp. sintenisii and, Clypeola jonthlaspi are frequently found in the association.

\section{Rubo sancti - Salicetum albae ass. nova (Table 3)}

Holotypus: relevé 25, Melendiz river, Cover $\% 85,50 \mathrm{~m}^{2}$ Lat: $38^{\circ} 15^{\prime} 15^{\prime \prime} \mathrm{N}$, Lon: $034^{\circ} 18^{\prime} 16^{\prime \prime} \mathrm{E}, 1189 \mathrm{~m}$.

This association flourishes on volcanic bedrock. Texture type of the soil is clay loam, containing $0.37-0.38 \%$ organic substances. The soil on which the association flourishes shows mildly alkaline reactions $(\mathrm{pH} 7.18-7.76) . \mathrm{CaCO}_{3}$ ratio is $0.52-0.59 \%$ and saturation is $55-56 \%$. Total salt content varies between 0.026 and 0.077 .

The association, which was detected where the slope was $2^{\circ}-5^{\circ}$, consists of tree, shrub and herb layers. Tree overlap is between $80-85 \%$ with an average height of $10 \mathrm{~m}$ and shrub overlap is between $45-50 \%$ with an average height of $1 \mathrm{~m}$. Grass overlap varies between $40-50 \%$ with an average height of $30-40 \mathrm{~cm}$. The association flourishes in a north-south direction throughout the Melendiz River.

The taxon Salix alba, which is an Euro-Siberian element, shows a wide distribution in many streams and rivers in Turkey. Rubus sanctus, Geranium purpureum, Veronica anagallis-aquatica subsp. oxycarpa, Nepeta nuda subsp. albiflora, Lepidium latifolium, Stellaria media subsp. media and, Lamium purpureum var. purperum are frequently found to participate in the association.

The Ihlara Valley lands can be divided into three groups in general; alluvial soils, brown soils and brown lime soils. There are also bare rocks and debris located in the area.

In the study area, riparian vegetation, which flourishes over alluvial soils, is found along the Melendiz River. Brown soils are the most widely distributed soil type group in the area. Tragacanth steppe vegetation flourishes over these calcareous Brown soils. Shrub vegetation, which flourishes over the hillsides in the area, generally flourishes over limeless brown soils, bare rock cracks and debris. 
Table 2. Astragaletum karamasici-microcephali ass. nova

\begin{tabular}{|c|c|c|c|c|c|c|c|c|c|c|c|c|}
\hline & Relevé No & 11 & 12 & 13 & 14 & 15 & 16 & 17 & 18 & 19 & 20 & \\
\hline & Size of plot $\left(\mathrm{m}^{2}\right)$ & 25 & 25 & 25 & 25 & 25 & 25 & 25 & 25 & 25 & 25 & \\
\hline & Parent rock & Vol & Vol & Vol & Vol & Vol & Vol & Vol & Vol & Vol & Vol & \\
\hline & Inclination $\left({ }^{\circ}\right)$ & 15 & 15 & 10 & 25 & 20 & 20 & 25 & 30 & 20 & 20 & \\
\hline & Altitude (m) & 1238 & 1235 & 1240 & 1254 & 1250 & 1244 & 1241 & 1292 & 1306 & 1301 & $\stackrel{\mathscr{U}}{\Xi}$ \\
\hline & Exposition & $\mathrm{NE}$ & $\mathrm{N}$ & $\mathrm{N}$ & $\mathrm{E}$ & $\mathrm{E}$ & $\mathrm{E}$ & $\mathbf{E}$ & $\mathrm{S}$ & SW & $\mathrm{W}$ & 必 \\
\hline & Coverage $(\%)$ & 80 & 80 & 75 & 80 & 85 & 85 & 85 & 85 & 75 & 70 & $\underline{2}$ \\
\hline \multicolumn{13}{|c|}{ LF } \\
\hline \multicolumn{13}{|c|}{ Differential and characteristic species of the association } \\
\hline $\mathrm{Ch}$ & Astragalus microcephalus & 4 & 5 & 5 & 5 & 4 & 4 & 5 & 4 & 5 & 4 & $\mathrm{~V}$ \\
\hline $\mathrm{H}$ & Astragalus karamasicus & 1 & + & & 1 & + & 1 & $\mathbf{1}$ & & 1 & 1 & IV \\
\hline \multicolumn{13}{|c|}{ Characteristic species of Phlomido armeniacae-Astragalion microcephali } \\
\hline $\mathrm{H}$ & Phlomis armeniaca & + & & 1 & & & & + & & + & & II \\
\hline \multirow{2}{*}{\multicolumn{13}{|c|}{$\begin{array}{l}\text { Onobrychido armenae-Thymetalia lecostomi karakter türleri } \\
\text { Paronychia kurdica subsp. kurdica var. }\end{array}$}} \\
\hline $\mathrm{H}$ & $\begin{array}{l}\text { Paronychia kurdica subsp. kurdica } \\
\text { kurdica }\end{array}$ & & & & + & & & & & & & III \\
\hline \multicolumn{13}{|c|}{ Characteristic species of Astragalo microcephali-Brometea tomentelli } \\
\hline $\mathrm{Th}$ & Xeranthemum аппиит & 1 & & + & 1 & 1 & + & & + & 1 & & IV \\
\hline $\mathrm{Ch}$ & Teucrium polium & + & & 1 & + & + & & 1 & + & & 1 & IV \\
\hline $\mathrm{H}$ & Poa bulbosa & & + & & 1 & & + & & + & & + & III \\
\hline $\mathrm{H}$ & Bromus tomentellus & & & + & + & + & + & + & + & & & III \\
\hline $\mathrm{H}$ & Cruciata taurica & & 1 & & + & & + & + & & 1 & & III \\
\hline Th & Medicago radiata & 1 & 1 & & & 1 & & & + & + & & III \\
\hline $\mathrm{Ch}$ & Acantholimon acerosum var. acerosum & + & & & & + & & + & & & & II \\
\hline G & Asphodeline taurica & & & + & & & + & + & & + & & II \\
\hline $\mathrm{H}$ & Euphorbia macroclada & & + & & & & & + & & + & & II \\
\hline $\mathrm{H}$ & Centaurea virgata & & & 1 & + & & 1 & + & & & & II \\
\hline $\mathrm{H}$ & Ajuga chamaepitys subsp. chia var. chia & & + & & & & + & + & + & & & II \\
\hline $\mathrm{H}$ & Onosma aucheranum & & + & & + & + & & & + & & & II \\
\hline $\mathrm{H}$ & Potentilla recta & & & + & & & + & + & & & & II \\
\hline $\mathrm{G}$ & Allium scorodoprasum subsp. rotundum & & + & & + & & & & & & + & II \\
\hline $\mathrm{H}$ & Salvia cryptantha & 1 & & & + & & & 1 & & 1 & & II \\
\hline $\mathrm{H}$ & Scabiosa argentea & & 1 & & 1 & + & & & 1 & & & II \\
\hline $\mathrm{H}$ & Stipa holosericea & + & & & + & & & + & & & & II \\
\hline $\mathrm{H}$ & $\begin{array}{l}\text { Gundelia tournefortii subsp. armata } \\
\text { Companions }\end{array}$ & & & & + & & & + & & & & I \\
\hline $\mathrm{H}$ & Marrubium globosum subsp. globosum & 1 & 1 & & + & 1 & & & 1 & & + & III \\
\hline Th & Ziziphora taurica subsp. taurica & 1 & & 1 & & & 1 & 1 & 1 & & + & III \\
\hline $\mathrm{Ch}$ & Thymbra sintenisii subsp. sintenisii & & & + & + & + & & + & & & + & III \\
\hline $\mathrm{H}$ & Anthemis pauciloba var. pauciloba & 1 & & + & & & + & + & + & & + & III \\
\hline Th & Androsace maxima & + & & + & & & + & + & + & & + & III \\
\hline $\mathrm{H}$ & Astragalus tigridis & + & & & + & & + & & + & + & & III \\
\hline Th & Clypeola jonthlaspi & 1 & & 1 & & 1 & 1 & & 1 & & 1 & III \\
\hline Th & Wiedemannia orientalis & + & + & & + & & & & + & + & & III \\
\hline $\mathrm{H}$ & Onobrychis elata & & 1 & 1 & & 1 & & + & 1 & & 1 & III \\
\hline Th & Minuartia hamata & + & & 1 & 1 & & + & & 1 & 1 & & III \\
\hline Th & Scandix stellata & & 1 & & + & 1 & & & 1 & + & + & III \\
\hline Th & Lamium macrodon & & + & & 1 & + & & + & & 1 & + & III \\
\hline Th & Trigonella coerulescens & & 1 & & + & & 1 & & & + & 1 & III \\
\hline $\mathrm{H}$ & Onosma angustissimum & & + & 1 & & + & 1 & & & + & & III \\
\hline Th & Alyssum strigosum subsp. strigosum & & 1 & & + & 1 & + & & 1 & & 1 & III \\
\hline Th & Ceratocephala falcatus & & 1 & & 1 & + & & + & + & & & III \\
\hline $\mathrm{H}$ & Ranunculus isthmicus subsp. stepporum & + & & & + & & + & + & & + & & III \\
\hline $\mathrm{H}$ & Echinops orientalis & + & + & & & + & & + & & & + & III \\
\hline Th & Malva neglecta & & + & & + & + & & $\mathbf{1}$ & + & & & III \\
\hline Th & Adonis aestivalis subsp. aestivalis & & + & & + & & + & & + & & 1 & III \\
\hline $\mathrm{H}$ & Haplophyllum vulcanicum & & & + & & & & + & & & + & II \\
\hline $\mathrm{H}$ & Lotus aegaeus & & & & + & & & & & + & & II \\
\hline $\mathrm{H}$ & Onobrychis tournefortii & & + & & + & & + & & & & + & II \\
\hline
\end{tabular}


Table 2. Continued

\begin{tabular}{|c|c|c|c|c|c|c|c|c|c|c|c|}
\hline Th Glaucium corniculatum subsp. refractum & + & & & + & & & + & & + & & II \\
\hline H Linaria corifolia & + & & & & + & & & + & & + & II \\
\hline H Silene alba subsp. divaricata & & + & & + & & & & & + & & II \\
\hline H Ononis spinosa subsp. leiosperma & + & & + & & + & & + & & & & II \\
\hline H Reseda lutea var. lutea & & + & & + & & + & & & & + & II \\
\hline H Ballota saxatilis subsp. saxatilis & + & & & & + & & + & & + & & II \\
\hline $\mathrm{H}$ Echium italicum & & + & & & + & + & & & + & & II \\
\hline Th Aethionema arabicum & & & + & & & & + & & & 1 & II \\
\hline H Alkanna pseudotinctoria & & + & + & & + & & & + & & & II \\
\hline Ch Astragalus ovalis & & & & + & + & & + & + & & & II \\
\hline Th Hordeum murinum subsp. murinum & 1 & & + & & & & + & 1 & & & II \\
\hline H Tragopogon pterodes & + & & & + & & & + & & & + & II \\
\hline G Iris sprengeri & + & & & + & & & + & & + & & II \\
\hline H Linum mucronatum subsp. armenum & & & + & & & & & + & + & & II \\
\hline H Torilis leptophylla & + & & 1 & + & & & & & + & & II \\
\hline Th Taeniatherum caput-medusae subsp. crinitum & & & & + & & & & + & + & & II \\
\hline H Dactylis glomerata subsp. hispanica & & + & & & + & & & + & & & II \\
\hline H Verbascum pycnostachyum & + & & & + & & & + & & & + & II \\
\hline Ch Thymus leucostomus var. leucostomus & & & & + & & + & & + & & + & II \\
\hline Ch Noaea mucronata subsp. mucronata & & & & & + & & + & & & & I \\
\hline Th Nigella arvensis var. glauca & + & & & & & & & & + & & I \\
\hline $\mathrm{H}$ Crambe orientalis subsp. orientalis & & & + & & & & & & & + & I \\
\hline
\end{tabular}

According to the relationship between plant associations in the study area and the soil texture types, the plant association belonging to the riparian vegetation flourishes best in clay-loam soils, the plant association that belongs to the tragacanth steppe vegetation flourishes best in loam soils, and the plant association that belongs to the shrub vegetation flourishes best in clay-loam and clay soils.

A large proportion of Turkish soil is alkaline. Soil $\mathrm{pH}$ classification is as follows; $\mathrm{pH}<4.5$ strong acid; 4.5-5.5 medium acid; 5.5-6.5 weak acid, 6.5-7.5 neutral; 7.5-8.5 weak alkali; pH>8.5 strong acid (Eyüpoğlu, 1999). When the analysed soils are considered according to this classification, soils with Astragaletum karamasicimicrocephali are weak alkali, Pistacio terebinthi- Celtidetum tournefortii are neutral, and Rubo sancti - Salicetum albae soils are weak alkali.

Electrical conductivity in soils is classified as; $\mathrm{EC}<2 \mathrm{dS} \mathrm{m}^{-1}$ non-saline; $2-4 \mathrm{dS} \mathrm{m}^{-}$ ${ }^{1}$ medium salinity; $8-15 \mathrm{dS} \mathrm{m}^{-1}$ highly saline and EC> $15 \mathrm{dS} \mathrm{m}^{-1}$ very highly saline (Richards, 1954). When the analysed soils are considered from an electrical conductivity point of view, the three soil syntaxa are all in the non-saline soil class.

Much of the soils in Turkey are lime rich. When classified according to lime content; lime content $<0-1 \%$ low lime; $1-5 \%$ limey; $5-15 \%$ medium degree lime; 15 $25 \%$ lime rich; $>25 \%$ very lime rich (Gedikoğlu, 1990). The lime content of the analysed soils varies widely. Generally, soils with Pistacio terebinthi-Celtidetum tournefortii are in the limeless and low lime content group, Rubo sancti-Salicetum albae soils have low lime content, whilst soils with Astragaletum karamasicimicrocephali are limey. 
Table 3. Rubo sancti - Salicetum albae ass. nova

\begin{tabular}{|c|c|c|c|c|c|c|c|c|c|c|c|c|}
\hline & Relevé No & 21 & 22 & 23 & 24 & 25 & 26 & 27 & 28 & 29 & 30 & \\
\hline & Size of plot $\left(\mathrm{m}^{2}\right)$ & 400 & 400 & 400 & 400 & 400 & 400 & 400 & 400 & 400 & 400 & \\
\hline & Parent rock & Vol & Vol & Vol & Vol & Vol & Vol & Vol & Vol & Vol & Vol & \\
\hline & Inclination $\left(^{\circ}\right)$ & 0 & 0 & 0 & 0 & $\mathbf{0}$ & 0 & 0 & 0 & 0 & 0 & \\
\hline & Altitude (m) & 1202 & 1202 & 1208 & 1208 & 1189 & 1194 & 1188 & 1190 & 1190 & 1192 & E \\
\hline & Exposition & $\mathrm{N}$ & $\mathrm{N}$ & $\mathrm{N}$ & $\mathrm{N}$ & $\mathbf{N}$ & $\mathrm{N}$ & $\mathrm{E}$ & $\mathrm{N}$ & NW & NW & 岁 \\
\hline & Coverage $(\%)$ & 80 & 85 & 80 & 85 & 85 & 85 & 85 & 80 & 75 & 80 & 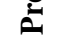 \\
\hline \multicolumn{13}{|c|}{$\mathbf{L F}$} \\
\hline \multicolumn{13}{|c|}{ Differential and characteristic species of the association } \\
\hline $\mathrm{Ph}$ & Salix alba & 5 & 5 & 5 & 5 & 5 & 5 & 5 & 5 & 5 & 5 & $\mathrm{~V}$ \\
\hline $\mathrm{Ph}$ & $\begin{array}{l}\text { Rubus sanctus } \\
\text { Characteristic species of Populion albae }\end{array}$ & 1 & & & 1 & 1 & 1 & 1 & 1 & & 1 & IV \\
\hline $\mathrm{Ph}$ & $\begin{array}{l}\text { Fraxinus angustifolia subsp. angustifolia } \\
\text { Populetalia albae karakter türleri }\end{array}$ & & & & & + & & + & & & & I \\
\hline $\mathrm{Ph}$ & $\begin{array}{l}\text { Ulmus minor subsp. minor } \\
\text { Characteristic species of Salici purpureae-Po }\end{array}$ & Iletea & nigra & ${ }^{+}$ & & + & & & & + & & II \\
\hline $\mathrm{Ph}$ & $\begin{array}{l}\text { Populus nigra subsp. nigra } \\
\text { Companions }\end{array}$ & & & & & & + & & & + & & I \\
\hline $\mathrm{Th}$ & Geranium purpureum & & + & & 1 & 1 & & + & 1 & & + & III \\
\hline $\mathrm{H}$ & Veronica anagallis-aquatica subsp. oxycarpa & 1 & & + & 1 & & + & & 1 & + & & III \\
\hline $\mathrm{H}$ & Nepeta nuda subsp. albiflora & & + & 1 & & + & + & & + & & 1 & III \\
\hline $\mathrm{H}$ & Lepidium latifolium & & + & + & + & 1 & & 1 & + & & & III \\
\hline $\mathrm{Th}$ & Stellaria media subsp. media & 1 & + & & 1 & 1 & & & 1 & + & & III \\
\hline $\mathrm{Th}$ & Lamium purpureum var. purperum & 1 & & + & 1 & & & & 1 & & + & III \\
\hline $\mathrm{H}$ & Vincetoxicum tmoleum & & & + & & + & & & + & & & II \\
\hline $\mathrm{H}$ & Tanacetum argyrophyllum var. argyrophyllum & & & + & & + & & & + & & & II \\
\hline $\mathrm{H}$ & Solanum dulcamara & + & & & & + & + & + & & & & II \\
\hline $\mathrm{H}$ & Asparagus verticillatus & & & + & & + & & & & + & & II \\
\hline $\mathrm{H}$ & Plantago major subsp. major & + & & & & & + & & 1 & & & II \\
\hline $\mathrm{H}$ & Astragalus ponticus & + & & + & & & & + & & & & II \\
\hline $\mathrm{Th}$ & Cuscuta campestris & & & & & + & & + & + & & & II \\
\hline $\mathrm{H}$ & Cichorium intybus & & & + & & + & + & & & & & II \\
\hline $\mathrm{H}$ & Calystegia sepium subsp. sepium & + & & + & & + & & + & & & & II \\
\hline $\mathrm{H}$ & Lysimachia vulgaris & & & & & + & & + & + & & & II \\
\hline $\mathrm{H}$ & Cucubalus baccifer & & + & & & & & & + & & + & II \\
\hline $\mathrm{H}$ & Typha latifolia & & & & & + & & + & + & & & II \\
\hline $\mathrm{Ph}$ & Tamarix smyrnensis & & & & + & & & + & + & & & II \\
\hline $\mathrm{H}$ & Dipsacus laciniatus & & & + & & & + & & & + & & II \\
\hline $\mathrm{H}$ & Rumex acetosella & & 1 & + & & & & + & & & & II \\
\hline Th & Geranium molle subsp. molle & & & & & + & & & + & + & + & II \\
\hline $\mathrm{H}$ & Lamium garganicum subsp. reniforme & & + & & & & + & 1 & & & & II \\
\hline Th & Geranium rotundifolium & & & + & & 1 & & & & & + & II \\
\hline $\mathrm{H}$ & Alliaria petiolata & + & & & & & & & & + & + & II \\
\hline $\mathrm{H}$ & Sparganium erectum subsp. neglectum & & + & & & + & & & & & + & II \\
\hline $\mathrm{H}$ & Lythrum salicaria & + & & & & & + & & & + & & II \\
\hline Th & Plantago scabra & & + & & + & & & & & + & & II \\
\hline $\mathrm{H}$ & Cynanchum acutum subsp. acutum & + & & & & + & & + & & + & & II \\
\hline $\mathrm{H}$ & Convolvulus arvensis & & & + & & + & & + & + & & & II \\
\hline $\mathrm{H}$ & Juncus gerardii subsp. gerardii & & + & & & + & & & & & + & II \\
\hline Th & Solanum nigrum subsp. nigrum & & + & & & & + & & + & & & II \\
\hline $\mathrm{Ph}$ & Elaeagnus angustifolia & & & & + & & & & & + & & I \\
\hline $\mathrm{H}$ & Prunella vulgaris & & + & & & & & + & & & & I \\
\hline Th & Koelpinia linearis & & & & & + & + & & & & & I \\
\hline $\mathrm{G}$ & Ornithogalum platyphyllum & & & & & + & & + & & & & I \\
\hline $\mathrm{H}$ & Scirpoides holoschoenus & & & + & & & & + & & & & I \\
\hline $\mathrm{H}$ & Pulicaria dysenterica & + & & & & + & & & & & & I \\
\hline $\mathrm{H}$ & Vincetoxicum fuscatum subsp. fuscatum & & & + & & & & + & & & & I \\
\hline $\mathrm{Ph}$ & Rubus discolor & & & + & & & & & & & + & I \\
\hline
\end{tabular}


Soils in Turkey tend to be poor in phosphorous. The classification of soils according to phosphorous content is; $\mathrm{P}_{2} \mathrm{O}_{5}<3 \mathrm{~kg} \mathrm{da}^{-1}$ very low; 3-6 low; 6-9 medium; 9-12 high and 12 very high (Eyüpoğlu, 1999). The Astragaletum karamasici - microcephali soils are very low in phosphorous, Rubo sancti Salicetum albae soils are low in phosphorous and Pistacio terebinthi-Celtidetum tournefortii soils are high in phosphorous.

The majority of soils in Turkey are sufficient or rich in potassium (Eyüpoğlu, 1999). The classification of soils according to potassium content is; $\mathrm{K}_{2} \mathrm{O}<20 \mathrm{~kg} \mathrm{da}^{-1}$ low; 20-30 medium; 30-40 sufficient and $\mathrm{K}_{2} \mathrm{O}>40$ high (Ülgen and Yurtsever, 1995). When the analysed soils are considered, all three soil syntaxa are variable in potassium content, with generally high potassium values.

Turkish soils are typically poor in organic matter (Eyüpoğlu 1999). When classified according to organic content; organic matter <1\% very low; $1-2 \%$ low; $2-3 \%$ medium; $3-4 \%$ good and organic matter $>4 \%$ high (Gedikoğlu, 1990). According to this, Astragaletum karamasici - microcephali and Rubo sancti - Salicetum albae soils are very low in organic content and Pistacio terebinthi - Celtidetum tournefortii soils are low in organic matter.

Analyses indicated that the soil parameters are in the optimal ratios for plant growth; no negations were observed. Accordingly, soil texture type and climate both play roles in the formation of plant associations in the study area (Table 4).

Table 4. Chemical analysis of the plant associations soils taken from the study area. C: clay, $L$ : loamy, CL: clay-loamy

\begin{tabular}{|c|c|c|c|c|c|c|c|c|c|c|}
\hline $\begin{array}{c}\text { Relevé } \\
\text { no }\end{array}$ & Associations & Texture & $\begin{array}{c}\text { Saturation } \\
(\%)\end{array}$ & $\underset{\mathrm{ds} / \mathrm{m}^{-1}}{\mathrm{EC}}$ & $\begin{array}{l}\text { Salt } \\
(\%)\end{array}$ & $\mathrm{pH}$ & $\begin{array}{c}\mathrm{CaCO}_{3} \\
(\%)\end{array}$ & $\begin{array}{c}\mathrm{P}_{2} \mathrm{O}_{5} \\
\left(\mathrm{~kg} / \mathrm{da}^{-1)}\right)\end{array}$ & $\begin{array}{c}\mathrm{K}_{2} \mathrm{O} \\
\left(\mathbf{k g} / \mathrm{da}^{-1}\right)\end{array}$ & $\begin{array}{c}\text { Organic } \\
\text { matter } \\
(\%)\end{array}$ \\
\hline 3 & \multirow{3}{*}{$\begin{array}{c}\text { Pistacio } \\
\text { terebinthi } \\
- \\
\text { Celtidetum } \\
\text { tournefortii }\end{array}$} & C & 98 & 1.234 & 0.077 & 6.59 & - & 18.70 & 259.81 & 8.60 \\
\hline 5 & & $\mathrm{CL}$ & 66 & 0.623 & 0.026 & 7.76 & 0.89 & 19.75 & 205.87 & 1.66 \\
\hline 9 & & $\mathrm{CL}$ & 61 & 0.707 & 0.028 & 6.89 & - & 14.77 & 26.84 & 1.51 \\
\hline 11 & \multirow{2}{*}{$\begin{array}{c}\text { Astragaletum } \\
\text { karamasici } \\
- \\
\text { microcephali }\end{array}$} & L & 47 & 0.673 & 0.020 & 8.01 & 1.19 & 0.88 & 116 & 0.38 \\
\hline 17 & & L & 44 & 0.630 & 0.018 & 7.99 & 1.33 & 0.72 & 68.49 & 0.48 \\
\hline 25 & \multirow{2}{*}{$\begin{array}{c}\text { Rubo } \\
\text { sancti } \\
- \\
\text { Salicetum } \\
\text { albae }\end{array}$} & $\mathrm{CL}$ & 56 & 0.052 & 0.020 & 7.18 & 0.59 & 5.14 & 71.31 & 0.38 \\
\hline 29 & & $\mathrm{CL}$ & 55 & 1.077 & 0.038 & 7.76 & 052 & 3.29 & 36.34 & 0.37 \\
\hline
\end{tabular}

The Ihlara Valley located in the Central Anatolia closed basin is one of the lowest precipitation areas in Turkey. The Melendiz River and its tributaries form the most important drainage system. There are mostly volcanic rocks with low water permeability located in Ihlara valley and the surrounding area. Water is found in three main areas as streams, stagnant water and thermal water (Gülkal, 2005). 
The Melendiz river, which is $60 \mathrm{~km}$ long (only $14 \mathrm{~km}$ of it flows through Ihlara Valley), is 1-2 meters wide at the steep entrance of Ihlara Valley, with the width reaching to 8-10 m after approximately $500 \mathrm{~m}$. The river forms a 2-3 m high waterfall at this point where the depth on the western side is sufficiently shallow for walking. This river furcates and forms gravelled islands where the slope of the land decreases. Melendiz becomes narrower at some places while wider at others until Belisirma, wherafter it expands towards Yaprakhisar after Kırkkazık (Gülkal, 2005).

As can be seen in the ombrothermic diagram from Aksaray station, the dry season starts at the end of May and lasts until November. Frost can be seen during January, February, and December in the study area (Fig. 3).

Researchers who study Mediterranean climates such as Emberger, Akman and Daget have divided the Mediterranean climate zone, which covers a very large area, into several sub-types of bioclimactic zones considering the gradual differentiations of the climate from coastal areas towards inner areas (Akman, 1981).

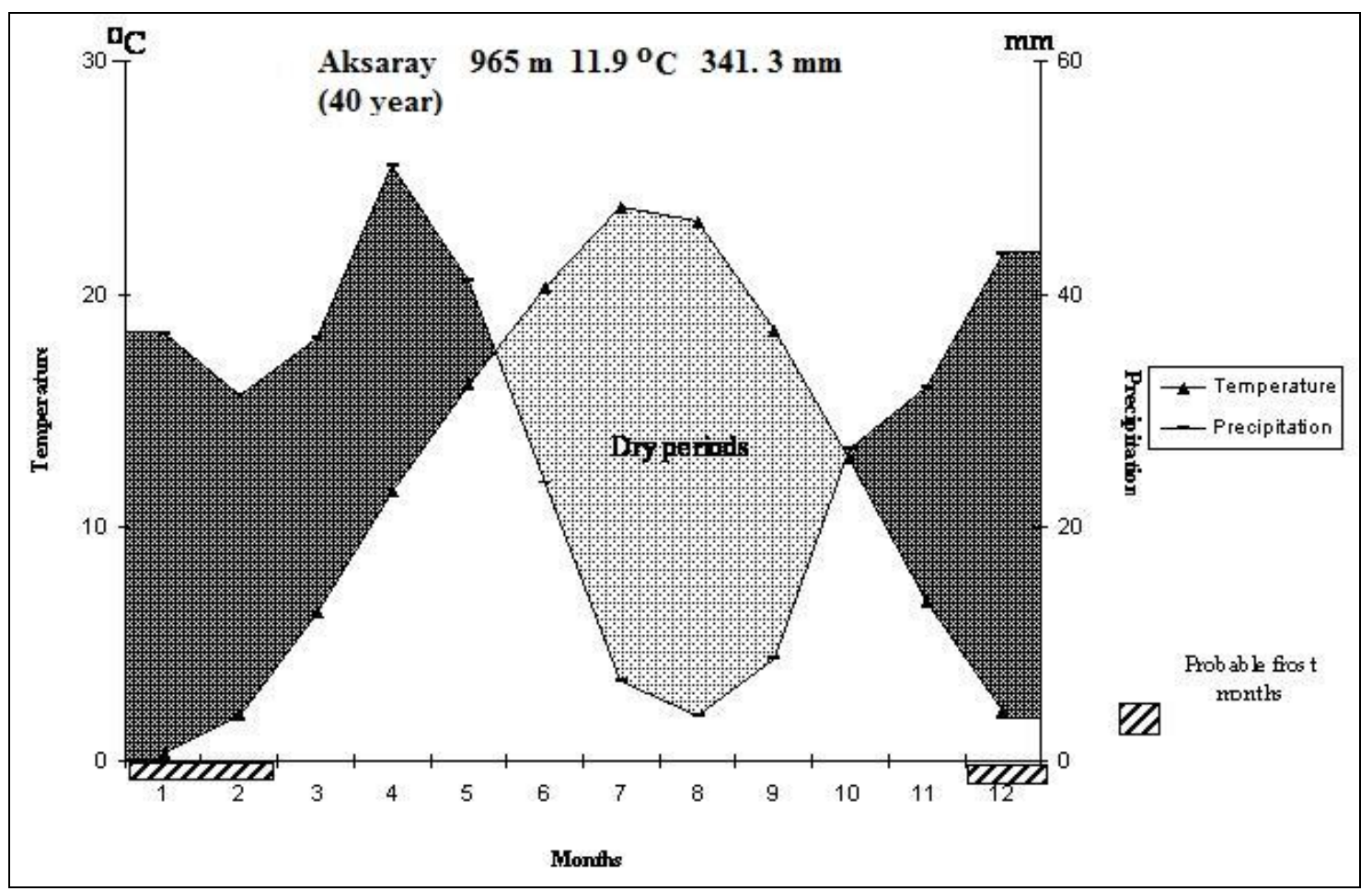

Figure 3. Ombrothermic diagram of Aksaray

The Emberger (1954) drought index $(\mathrm{S}=\mathrm{PE} / \mathrm{M})$ at the Aksaray station, which includes Ihlara valley, is 1.12 . As the $S$ value is below 5 , the study area is within the Mediterranean climate, indicating the presence of a minimum summer rainfall and a significant summer drought. The Emberger (1954) precipitation-temperature ratio (Q) gains an ecological importance when considered along with the average minimum temperature of the coldest month $(\mathrm{m})$. When these two values are applied to the Emberger (1954) diagram, the following results emerge for the study area (Table 6). 
Table 6. Bioclimatic analyeses of Aksaray Station [P: mean annual precipitation in mm; $M$ : mean maximum temperature $\left({ }^{\circ} \mathrm{C}\right)$ for the hottest month; $m$ : mean minimum temperature $\left({ }^{\circ} \mathrm{C}\right)$ for the coldest month; Q: Emberger's pluviometric quotient: 2000 P/M2-m2; PE: Summer rainfall; S: Emberger's index of xericity; Sp: Spring; W: Winter; A: Autumn; Sm: Summer, medit.: Mediterranean.]

\begin{tabular}{|c|c|c|c|c|c|c|c|c|c|}
\hline Station & $\begin{array}{c}\text { Altitude } \\
(\mathbf{m})\end{array}$ & $\begin{array}{c}\mathbf{P} \\
(\mathbf{m m})\end{array}$ & $\mathbf{P E}$ & $\mathbf{S}$ & $\begin{array}{c}\mathbf{M} \\
\left({ }^{\circ} \mathbf{C}\right)\end{array}$ & $\begin{array}{c}\mathbf{m} \\
\left({ }^{\circ} \mathbf{C}\right)\end{array}$ & $\mathbf{Q}$ & $\begin{array}{c}\text { Rainfall } \\
\text { regime }\end{array}$ & $\begin{array}{c}\text { Bioclimate } \\
\text { type }\end{array}$ \\
\hline Aksaray & 965 & 341.3 & 34.3 & 1.12 & 30.6 & -3.8 & 34.3 & Sp.W.A.Sm. & $\begin{array}{c}\text { Semiarid cold } \\
\text { medit. }\end{array}$ \\
\hline
\end{tabular}

Accordingly, the Aksaray station, which includes the study area, is classified under "semi-arid Mediterranean climate with cold winters" conditions and shows the characteristics of the East Mediterranean precipitation regime second variant (Sp.W.A.Sm.).

\section{Discussion}

In the Ihlara Valley, three plant associations were detected belonging to shrub, tragacanth steppe and riparian vegetation types. Shrub vegetation flourishes in the lower slopes of the valley, tragacanth steppe vegetation flourishes in the upper slopes of the valley and riparian vegetation flourishes along the Melendiz riverside, which flows through the middle of the valley.

Amongst the natural vegetation of the Ihlara Valley, shrub vegetation formed by two tree species in the form of shrub including Celtis tournefortii and Pistacia terebinthus subsp. terebinthus, as well as Colutea cilicica and Pyracantha coccinea taxa is seen on the valley slopes at altitudes of 1205 and $1290 \mathrm{~m}$ between Ihlara town and Belisirma village along $14 \mathrm{~km}$ of valley, especially on the slopes facing east and northeast. These are pure associations in which Celtis tournefortii determines the physiognomy and dominates the vegetation. As the west-facing slopes of the valley are steep, shrub vegetation in these areas are quite weak (Fig. 4).

The high number of types with less repetition in the Pistacio terebinthi-Celtidetum tournefortii association identified in the shrub vegetation indicates an anthropogenic effect or negative living conditions for the plants on the valley slopes.

Riparian vegetation was detected in the Ihlara Valley starting from Ihlara town, which is at the southern end of the valley, and extending for $14 \mathrm{~km}$ until Selime town between altitudes of 1189 and $1202 \mathrm{~m}$ throughout the Melendiz River flowing at the bottom of the valley. Rubo sancti - Salicetum albae syntaxa were identified from this vegetation in which Salix alba was dominant (Fig. 5).

Following a literature search (Braun-Blanquet et al., 1952; Zohary, 1973; Demiriz, 1993; Ketenoglu et al., 2010) performed for these two syntaxa identified at the study area, it was found that these two syntaxa are new to the world of science as they have not been identified previously.

Tragacanth steppe vegetation dominated by Astragalus microcephalus in the Ihlara Valley flourishes for $1 \mathrm{~km}$ from Belisirma town to Selime town at the upper slopes between altitudes of $1124 \mathrm{~m}$ and $1306 \mathrm{~m}$. Astragaletum karamasici - microcephali syntaxa was identified from this vegetation type (Fig. 6). 


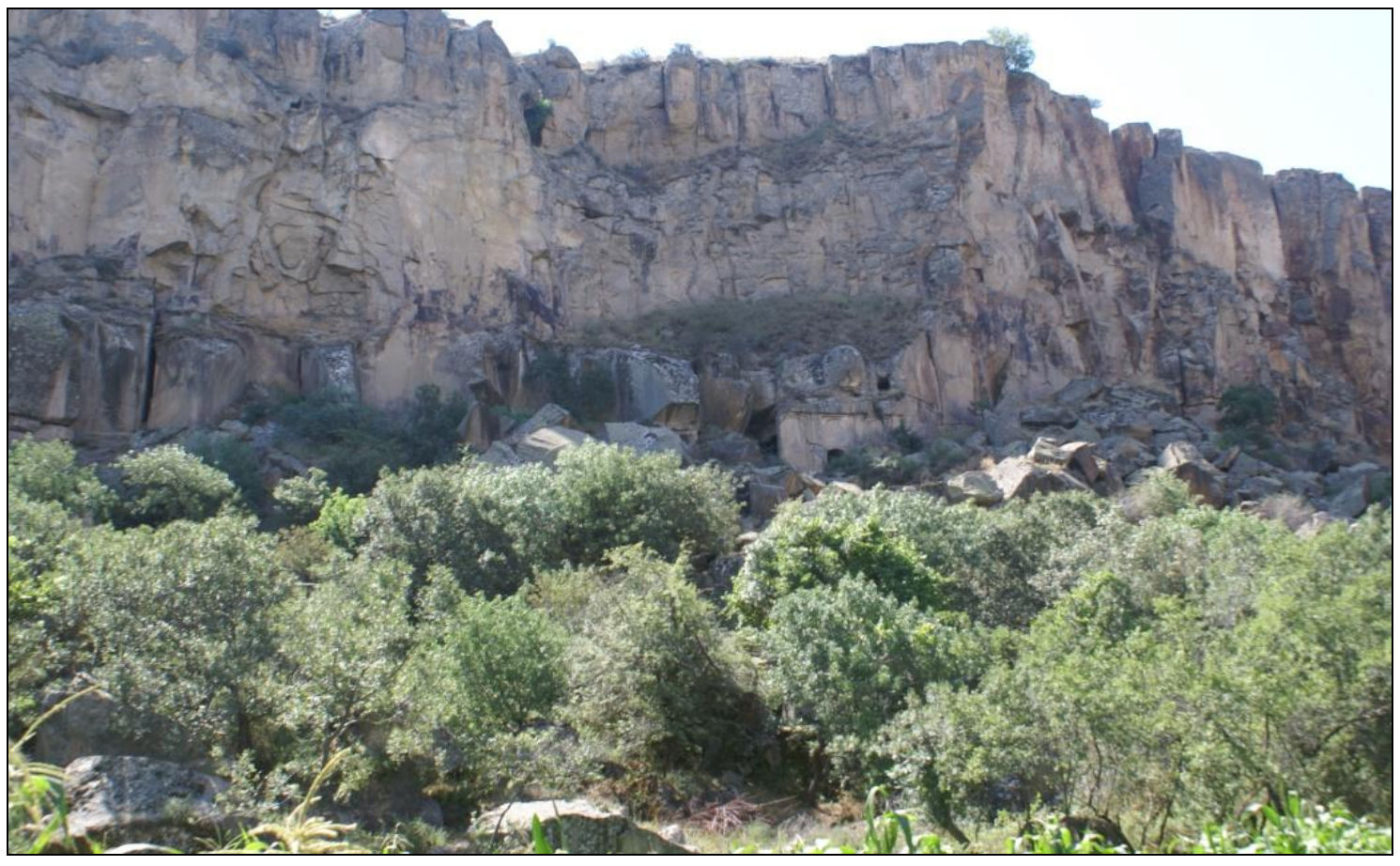

Figure 4. A view of Pistacio terebinthi-Celtidetum tournefortii

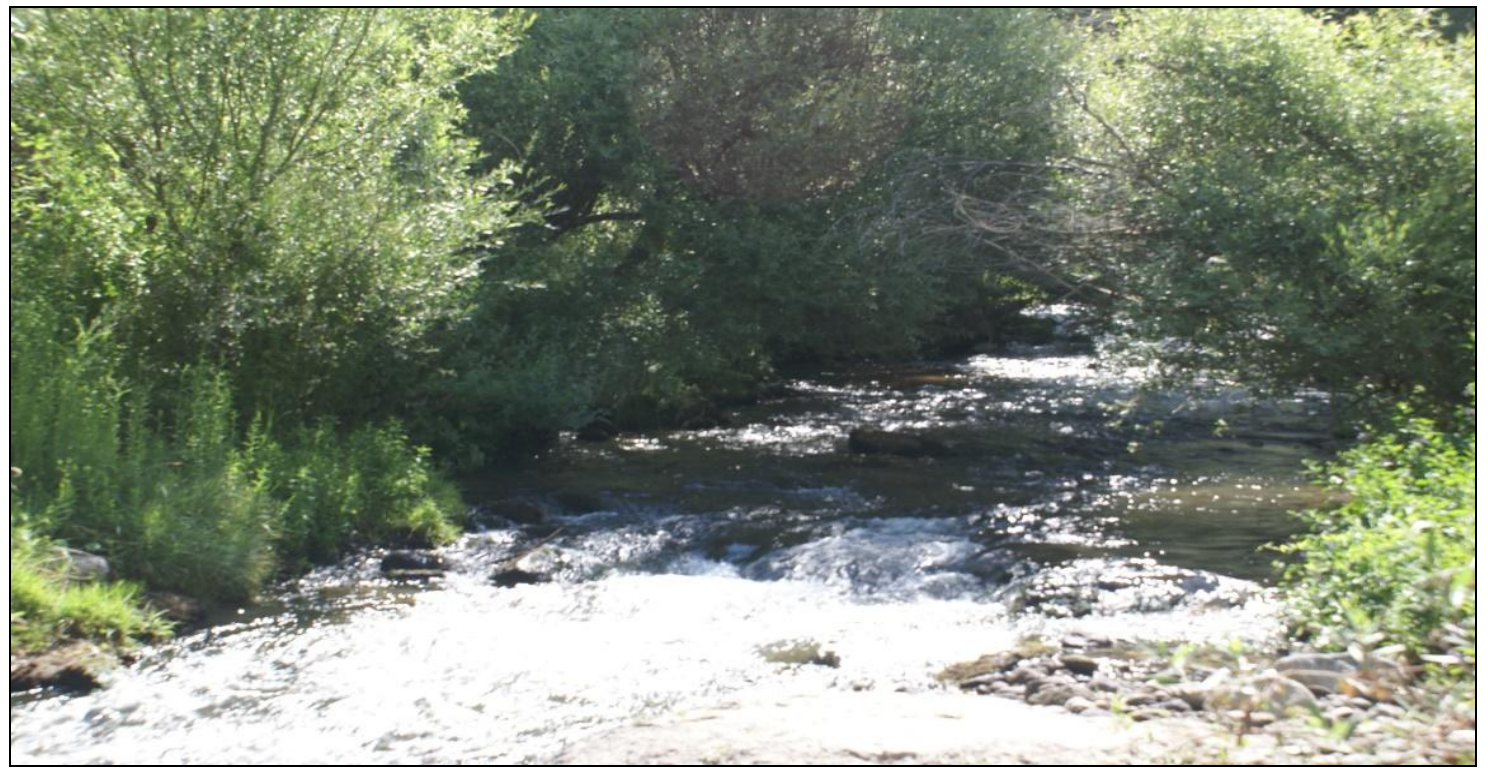

Figure 5. A view of Rubo sancti-Salicetum albae

Astragalus microcephalus has been determined as the dominant type for syntaxa identified in various phytosociological studies carried out in different parts of Turkey and also named in the co-dominancy of various taxa. Comparing the syntaxa identified in Ihlara Valley with the syntaxa identified in the studies carried out in Çubuk Dam (Çetik, 1963), Beynam Forest (Akman, 1972), Serpentine formation on Kırıkkale, Kalecik and, Elmadağ (Kılınç, 1974), Region among Beypazarı, Karaşar and Nallıhan 
(Akman, 1974), Kepekli Pass (Çetik and Düzenli, 1975), Hasan Mountain (Düzenli, 1976), Işık Mountain (Akman, 1976), Ayaş Mountain (Akman and Ketenoğlu, 1976), Semen Mountain (Akman et al., 1983), transition zone between Inner Anatolia and Western Black Sea (Kılınç, 1985), Aydos Mountains (Akman, 1990), Karadağ Mountain (Ocakverdi and Ünal, 1991), Anti-Taurus Mountains (Akman et al., 1991), Kızılören, Çal and Loras Mountains (Tatlı et al., 1994), Surrounding area between Ayaş-Polatlı and Beypazarı (Aydoğdu et al., 1994), Region between Alaçam-Gerze and Boyabat-Durağan (Özen and Kılınç, 1995), Dinek Mountain (Hamzaoğlu, 2005), Karaömer Mountain (Cansaran et al., 2010), similarity ratio was below 50\% according to the similarity formula described by Sørensen (1948). Within the light of these results, these syntaxa identified from the study area should be considered as new to the world of science.

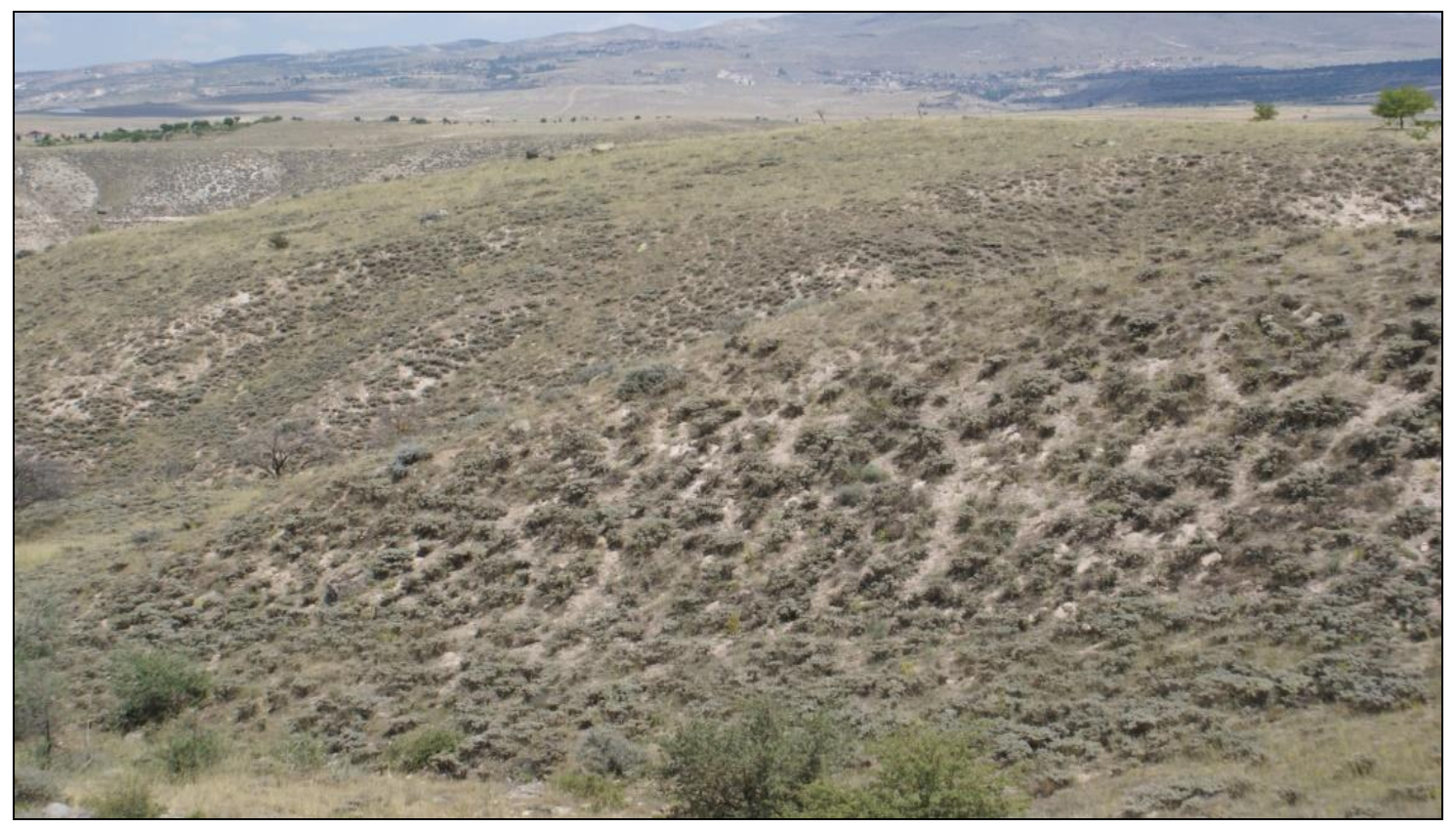

Figure 6. A view of Astragaletum karamasici-microcephali

Ihlara Valley has been a preferred settlement area since the earliest times due to its suitable climate conditions. This has led to anthropogenic influences, along with climate factors, directing the vegetation of the valley to its present condition. For example, along with many Populus nigra, Juglans regia and Robinia pseudoacacia planted by people along the the Melendiz riverside, there are also corn, wheat and barley planted by the settlers together with field weeds in the small plains in the valley.

In such a deep and narrow canyon as the Ihlara Valley, strong isolation conditions lead to the formation of a microclimate field. This prevents the areal expansion of the plants by active or passive distribution and also causes the inner valley to be affected less by the long dry summer conditions of Central Anatolia (Tanyolaç, 1995).

Examining the chorology of the taxa in the plant associations identified in Ihlara Valley, despite being in Irano-Turanian phytogeographical region, Euro-Siberian elements are very common in the plant associations (Pistacio terebinthi-Celtidetum tournefortii ve Rubo sancti - Salicetum albae) found on the slopes of the inner valley 
and at the Melendiz riverside at the bottom of the valley. Additionally, Irano-Turanian elements are very common in the plant association (Astragaletum karamasici microcephali) identified at the upper parts of the valley. This is a result of the microclimatic conditions provided by the geomorphological structure of the Ihlara Valley (Fig. 7).

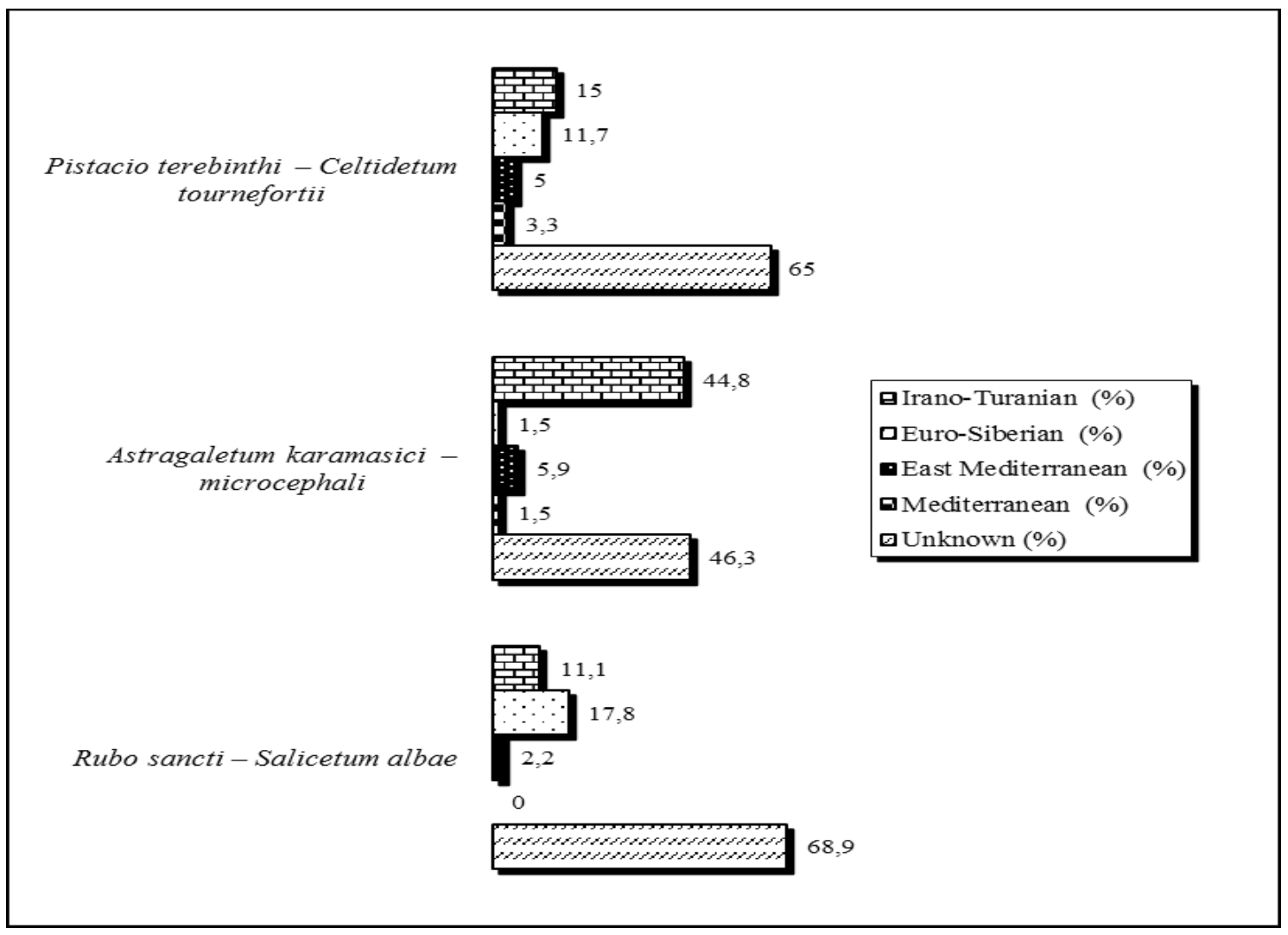

Figure 7. Chorology of taxa in plant associations

Examining the distribution of taxa identified in plant associations in Ihlara Valley according to Raunkiaer (1934), it was observed that, in general, hemicriptophytes ranked first in all three syntaxa. Phanerophytes were ranked second in Pistacio terebinthi-Celtidetum tournefortii and Rubo sancti - Salicetum albae syntaxa, in which Euro-Siberian phytogeographical elements characterized by a mesophytic vegetation containing abundant amounts of the phanerophytes and hemicriptophytes are very common. Terophytes and chamaephytes were ranked second in Astragaletum karamasici - microcephali syntaxa, which contains Irano-Turanian phytogeographical elements characterized by a xerophytic vegetation with abundant amounts of chamaephytes and hemicriptophytes. Conclusively, these life form distributions in the syntaxa are in compliance with their vegetation type and phytogeographical regions (Fig. 8).

In Ihlara Special Environmental Conservation Zone, habitat classes were determined according to the criteria determined by European Nature Information System (EUNIS) (Anonymous, 2004). The names of the seven identified habitat classes and their locations at the area are given in Fig. 9. 


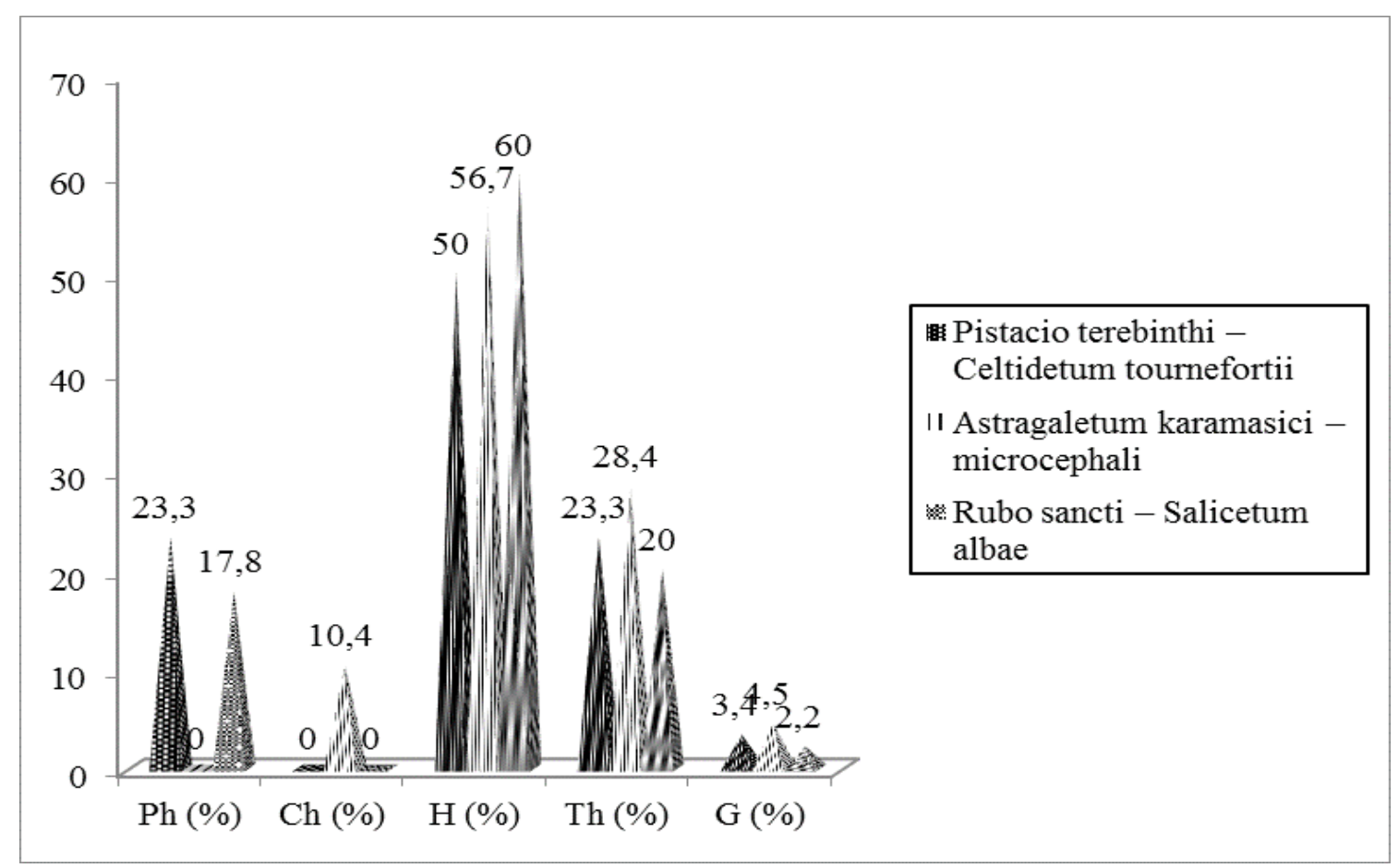

Figure 8. Life forms of plant associations

The syntaxonomy of the plant associations identified in this study in Ihlara Special Environmental Conservation Zone within Aksaray province is as follows:

$\underline{\text { Shrub vegetation }}$

Class: Quercetea pubescentis (Oberd. 1948) Doing Kraft 1955

Order: Querco cerridis - Carpinetalia orientalis Quézel, Barbero \& Akman 1980

Alliance: Melitto albidae - Quercion frainetto Barbero \& Quézel, 1976

Association: Pistacio terebinthi-Celtidetum tournefortii ass.nova

Tragacanth steppe vegetation

Class: Astragalo microcephali - Brometea tomentelli Quézel 1973

Order: Onobrychido armenae - Thymetalia leucostomi Akman, Ketenoğlu, Quézel 1985

Allliance: Phlomido armeniacae - Astragalion microcephali Akman, Ketenoğlu, Quézel, Demirörs 1984

Association: Astragaletum karamasici-microcephali ass.nova

$\underline{\text { Riparian vegetation }}$

Class: Salici purpureae-Populetea nigra Rivas-Martínez \& Cantó ex Rivas-Martínez, Báscones, T.E. Díaz, Fernández-González \& Loidi 1991

Order: Populetalia albae Br.-Bl. ex Tchou 1948

Alliance: Populion albae Br.-Bl. ex Tchou 1948

Association: Rubo sancti - Salicetum albae ass.nova 


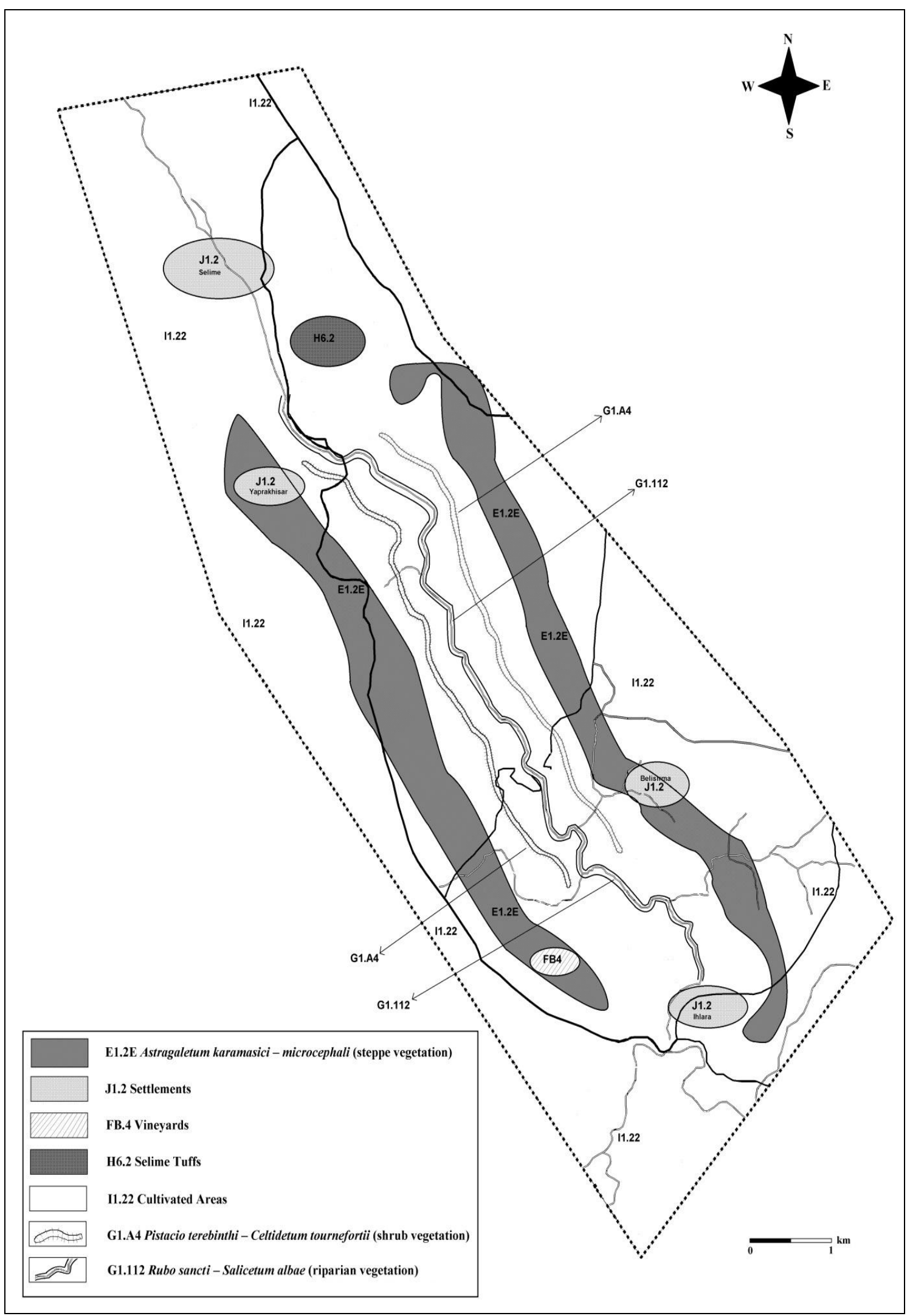

Figure 9. Eunis habitat classification of Ihlara Valley 
Acknowledgements. We are indebted to "General Directorate of Natural Assets Protection" for support. The authors thank Prof. Dr. A. Selçuk Ertekin (Dicle University) for help to identify the taxa, Assoc. Prof. Dr. Ali Volkan Bilgili (Harran University) and Dr. Sadık Yetim (Soil-Water Researches and Agricultural Research Institute) for help to interpret soil analysis, Abdi Seyarc1 and Onur Baysoy (Harran University) for help to field survey and Mehmet Fatih Özdemir (Harran University) for help to map arrangement.

Change history: The online version of this paper was modified on $9^{\text {th }}$ March 2017 . In the original version the syntaxon name for the riparian vegetation was written as Rubo sanctodis - Salicetum albae, but according to "International Code of Phytosociological Nomenclature $3^{\text {rd }}$ Edition" (Weber et al., 2000) it should have been written as Rubo sancti-Salicetum albae.

\section{REFERENCES}

[1] Akman, Y., Daget, P.H. (1971): Quelques aspects synoptiques des climates de la Turquie. - Bulletin de la Société Longuedocienne de Géographie 5(3): 269-300.

[2] Akman, Y. (1972): The vegetation of Beynam forest. - Communications de la Faculté des Sciences de 1'Université d'Ankara C(16): 31-53.

[3] Akman, Y. (1974): Etude phyto-ecologique de la region de Beypazar1-Karaşar et Nallihan. - Communications de la Faculté des Sciences de l'Université d'Ankara C(18): $51-113$

[4] Akman, Y. (1976): Etude phytosociologique du Masif D'ışık. - Communications de la Faculté des Sciences de l'Université d'Ankar, C2(20): 1-30.

[5] Akman, Y., Ketenoğlu, O. (1976): The phytosociological and pytoecological investigations on the Ayaş mountains. - Communications de la Faculté des Sciences de l'Université d'Ankara C2(20): 1-43.

[6] Akman, Y. (1981): Climats et bioclimats en Turquie. - Ecologia Mediterranea 8(1/2): 7387.

[7] Akman, Y., Yurdakulol, E., Demirörs, M. (1983): A phytosociological research on the vegetation of the Semen mountains (Bolu). - Communications de la Faculté des Sciences de l'Université d'Ankara C(1): 71-86.

[8] Akman, Y., Ketenoğlu, O., Quézel, P., Demirörs, M. (1984): A syntaxonomic study of steppe vegetation in Central Anatolia. - Pyhtocoenologia 12(4); 563-584.

[9] Akman, Y., Ketenoğlu, O., Quézel, P. (1985): A new syntaxon from Central Anatolia. Ecologia Mediterranea 11(2/3): 111-121.

[10] Akman, Y. (1990): Etude de la vegetation steppique des Montagnes d'Aydos situee au nord ouest d'Ankara. - Ecologia Mediterranea 16: 223-230.

[11] Akman, Y., Quézel, P., Barbero, M., Ketenoğlu, O., Aydoğdu, M. (1991): La végétation des steppes, pelouses écorchées et a xérophytes épineux de l'Antitaurus dans la partie sudouest de l'Anatolie. - Phytocoenologia, 19(3): 391-428.

[12] Akman, Y. (1995): Türkiye Orman Vejetasyonu. - Ankara Üniversitesi Fen Fakültesi Yayın1, Ankara.

[13] Akman, Y. (1999): İklim ve Biyoiklim (Biyoiklim Metodları ve Türkiye İklimleri). Kariyer Matbaacilık, Ankara.

[14] Anonymous. (2004): http://eunis.eea.europa.eu/upload/EUNIS_2004_list.pdf ANNEX 1 Index numbers and names of all EUNIS Habitats 2004.

[15] Anonymous. (2010): Aksaray meteoroloji istasyonu rasat verileri. - DMI Genel Müdürlüğü Araştırma ve Bilgi İşlem Dairesi Başkanlığı Arşivi, Ankara.

[16] Anonymous. (2012): Aksaray İli Çevre Durum Raporu-2011. - Aksaray Valiliği Çevre ve Şehircilik İl Müdürlüğü, Aksaray.

[17] Avcı, M. (2005): Çeşitlilik ve endemizm açısından Türkiye'nin bitki örtüsü. - İstanbul Üniversitesi Coğrafya Dergisi 13: 27-55. 
[18] Aydoğdu, M., Akman, Y., Quezel, P., Barbero, M., Ketenoğlu, O., Kurt, L. (1994): Syntaxonomic analysis of gypsaceous vegetation of the surrounding area between AyaşPolatlı and Beypazarı (Ankara, Turkey). - Ecologia Mediterranea 20(3/4): 9-19.

[19] Aydoğdu, M., Ketenoğlu, O., Hamzaoğlu, E. (1999): New syntaxa from Cappadocia (Kırşehir-Türkiye). - Israel Journal of Plant Sciences 47: (123-129).

[20] Aydoğdu, M., Hamzaoğlu, E., Kurt, L. (2002): New halophytic syntaxa from Central Anatolia (Turkey). - Israel Journal of Plant Sciences 50: 313-323.

[21] Aydoğdu, M., Kurt, L., Hamzaoğlu, E., Ketenoğlu, O., Cansaran, A. (2004): Phytoosociological studies on salty steppe communities of the Central Anatolia, Turkey. Israel Journal of Plant Sciences 52: 71-79.

[22] Barbero M., Quezel, P. (1976): Les groupements forestiers de Grèce centro-méridionale. Ecologia Mediterranea, 2: 1-86.

[23] Başköse, İ., Dural, H. (2011): The flora of Hasan (Aksaray Region, Turkey) Mountain. Biodicon 4(2): 125-148.

[24] Baysal, A. (2003): Syntaxonomical analysis of the steppe vegetation in region among Aksaray-Niğde and Kırşehir "Ph.D. Thesis". - Ankara University Graduate School of Natural and Applied Sciences Deparment of Biology, Ankara.

[25] Bouyoucos, G.J. (1936): Directions for making mechanical analysis of soils by the Hydrometer method. - Soil Science, 4: 225-228.

[26] Braun-Blanquet, J. (1932): Plant Sociology: The Study of Plant Communities (Translated, Revised and Edited by G.D.Fuller and H.S.Conard). - McGraw-Hill Book, New York.

[27] Braun-Blanquet, J., Roussine, M.N., Négre, R. (1952): Les groupements végétaux de la France Méditerranéenne. - Centre National De La Recherche Scientifique "Service de la Carte des Groupements Végétaux", Montpellier.

[28] Cansaran, A., Kaya, Ö.F., Ertekin, A.S., Ketenoğlu, O. (2010): A phytosociological study on Karaömer Mountain of North Anatolia (Amasya, Turkey). - Acta Botanica Gallica, 157(1): 65-88.

[29] Çetik, R. (1963): Çubuk barajının vejetasyonu. - İstanbul Üniversitesi Fen Fakültesi Mecmuas1, BXXVIII (3-4): 109-138.

[30] Çetik, R., Düzenli, A. (1975): Kepekli boğazı Atatürk ormanı ağaçlandırma alanının fitososyolojik ve fitoekolojik incelenmesi. - Orman Araştırma Enstitüsü Dergisi 21(2): 20-44.

[31] Çetik, R. (1985): Türkiye Vejetasyonu: I. İç Anadolu'nun Vejetasyonu ve Ekolojisi. Selçuk Üniversitesi Basımevi, Konya.

[32] Çırpıcı, A. (1987): Türkiye'nin flora ve vejetasyonu üzerine çalışmalar. - Doğa TU Botanik Dergisi 11(2): 217-232.

[33] Davis, P.H. (1965-1985): Flora of Turkey and the East Aegean Islands Vol. 1-9. Edinburg University Press, Edinburgh.

[34] Davis, P.H., Mill, R.R., Tan, K. (1988): Flora of Turkey and the East Aegean Islands Vol. 10 (Supplement). - Edinburg University Press, Edinburgh.

[35] Demiriz, H. (1993): An Annotated Bibliography of Turkish Flora and Vegetation. - The Scientific and Technological Research Council of Turkey, Ankara.

[36] Düzenli A. (1976): Hasan Dă̆ı'nın bitki ekolojisi ve bitki sosyolojisi yönünden araştırılmas1. - Orman Araştırma Enstitüsü Dergisi, 22(2): 7-53.

[37] Emberger L. (1954): Une classification biogéorgaphique des climats. - Recueil Travaux Laboratoire Botanique Géologie Zoologie Faculté Science Université Montpellier Série Botanique 7: 3-43.

[38] Eyüpoğlu, F. (1999): Türkiye Topraklarının Verimlilik Durumu. - Toprak ve Gübre Araştırma Enstitüsü Yayınları, Ankara.

[39] Frey, W., Lösch, R. (1998): Lehrbuch der Geobotanik: Pflanze und Vegetation in Raum und Zeit. - Gustav Fischer, Stuttgart. 
[40] Gedikoğlu, I. (1990): Toprak Verimliliğinin Tayininde Kullanılan Laboratuvar Analiz Yöntemleri. Şanlıurfa Araştırma Enstitüsü Müdürlüğü Yayınları, Şanlıurfa.

[41] Gülkal, Ö. 1999: Determination of criteria in the elaboration of planing procedures addressing protection use equilibrium an example: Ihlara (Cappadocia) specially protected area and its close vicinity "Ph.D. Thesis". - Department of Landscape Architecture Institute and Applied Sciences University of Çukurova, Adana.

[42] Gülkal, Ö. (2005): Ihlara Özel Çevre Koruma Bölgesi. - Çevre ve Orman Bakanlığı Özel ve Çevre Koruma Kurumu Başkanlığı Yayını, Ankara.

[43] Güner, A., Özhatay, N., Ekim, T., Başer, K.H.C. (2000): Flora of Turkey and the East Aegean Islands Vol. 11 (Supplement 2). - Edinburgh University Press, Edinburgh.

[44] Günal, N. (2013): Türkiye'de İklimin Doğal Bitki Örtüsü Üzerindeki Etkileri. - Acta Turcica 5(1): 1-22.

[45] Hamzaoğlu E. (2005): The Steppe Vegetation of Dinek Mountain (Kırıkkale). - Gazi University Journal of Science 18(1): 1-15.

[46] Ketenoğlu, O., Aydoğdu, M., Kurt, L., Akman, Y., Hamzaoğlu, E. (2000): Syntaxonomic research on the gypsicole vegetation in Cappadocia, Turkey. - Israel Journal of Plant Sciences 48: 121-128.

[47] Ketenoğlu, O., Aydoğdu, M., Kurt, L., Hamzaoğlu, E., Tuğ, G. N., Aslantürk, N. (2008): New syntaxa from stepe vegetation in Cappadocia, Turkey. - Anadolu University Journal of Science and Technology 9: 123-133.

[48] Ketenoglu, O., Tuğ, G.N., Bingöl, Ü., Geven, F., Kurt, L., Güney, K. (2010): Synopsis of syntaxonomy of Turkish forests. - Journal of Environmental Biology, 31: 71-80.

[49] Kilınç, M. (1974): Kırıkkale-Kalecik ve Elmadağ arasındaki serpantin formasyonunun vejetasyonu üzerinde ekolojik ve sosyolojik bir araştırma. - Bitki 1(4): 479-521.

[50] K1lıç, M. (1985): İç Anadolu-Batı Karadeniz geçiş bölgesinde Devrez çayı ile Kızılırmak nehri arasında kalan bölgenin vejetasyonu. - Doğa Bilim Dergisi, 9(2): 315357.

[51] Ocakverdi, H., Ünal, A. (1991): Karadağ'ın (Karaman) bitki sosyolojisi ve ekolojisi yönünden incelenmesi. - Turkish Journal of Botany 15: 79-106.

[52] Olsen, S.R., Cole C.V., Watanabe, F.S., Dean, L.A. (1954): Estimation of Available Phosphorus in Soils by Extraction with Sodium Bicarbonate. - United States Department of Agriculture, Washington DC.

[53] Özen, F., Kılınç, M. (1995): Alaçam-Gerze ve Boyabat-Durağan arasında kalan bölgenin vejetasyonu: I-Maki, frigana, dere ve step vejetasyonları. - Turkish Journal of Botany 19: 65-86.

[54] Quézel, P. (1973): Contribution a l'etude phytocoenologique du masif du Taurus. Phytocoenologia 1(2): 131-222.

[55] Quézel P., Barbero M., Akman, Y. (1980): Contrubition a l'etude de la vegetation forestiere d'Anatolie septentrionale. - Phytocoenologia 8(3/4): 365-519.

[56] Raunkiaer, C. (1934): The Life Forms of Plants and Statistical Plant Geography. Clarendon Press, Oxford.

[57] Richards, L.A. (ed.) (1954): Diagnosis and Improvement of Saline and Alkali Soils. United States Department of Agriculture, Washington DC.

[58] Rivas-Martínez, S., Báscones, J.C., Diaz, T.E., Fernández-González, F., Loidi, J. (1991): Vegetación del Pirineo Oriental y Navarra. - Itinera Geobotanica 5: 5-456.

[59] Schollenberger, C.J., Simon, R.H. (1945): Determination of exchange capacity and exchangeable bases in soil-Ammonium acetate method. - Soil Science, 59(1): 13-24.

[60] Sherrod, L.A., Dunn, G., Peterson, G.A., Kolberg, R.L. (2002): Inorganic carbon analysis by modified pressure-Calcimeter method. - Soil Science Society of America Journal, 66: 299-305.

[61] Sørensen, T. (1948): A method of establishing groups of equal amplitude in plant sociology based on similarity of species content, and its application to analyses of the 
vegetation on Danish commons. Det Kongelige Danske Videnskabernes Selskabs Biologiske Skrifter 5(4): 1-34.

[62] Tanker, N., Koyuncu, M., Kurucu, S. (1989): Ürgüp, Göreme and Ihlara Plants and Landscape. - Nurol Matbaacilik, Ankara.

[63] Tanyolaç J., Çelik N., Olgun K., Koca S., Gencer L., Dönmez, E. (1995): Ihlara Vadisi'nin Flora, Fauna, Ekolojik Varlıklarının Tesbiti ve Korunması. - Devlet Planlama Teşkilatı Raporu, Ankara.

[64] Tatlı, A., Eyce, B., Serin, M. (1994): Kızılören, Çal ve Loras Dağları (Konya) vejetasyonu. - Turkish Journal of Botany 18: 267-288.

[65] Ülgen, N., Yurtsever, N. (1995): Türkiye Gübre ve Gübreleme Rehberi. Toprak ve Gübre Araştırma Enstitüsü Yayınları, Ankara.

[66] Walkley, A., Black, I.A. (1934): An examination of the Degtjareff method for determining soil organic matter and a proposed modification of the chromic acid titration method. - Soil Science, 37: 29-38.

[67] Walter, H. (1962): Anadolunun Vejetasyon Yapıs1 "Die Vegetationsverhältnisse Anatoliens" (Translated S. Uslu). - İstanbul Üniversitesi Orman Fakültesi Yayını, İstanbul.

[68] Walter, H. (1972): İç Anadolu Step Problemi “Anatoliens Steppenprobleme” (Translated S. Uslu). - İstanbul Üniversitesi Orman Fakültesi Yayını, İstanbul.

[69] Weber, H.E., Moravec J., Theurillat J.P. (2000): International code of phytosociological nomenclature 3rd edition. - Journal of Vegetation Science 11: 739-768.

[70] Zohary, M., (1973): Geobotanical Foundations of the Middle East, 1-2. - Gustav Fischer Verlag, Stuttgart. 